PHYSICAL REVIEW D 93, 014026 (2016)

\title{
Global analysis of nuclear parton distribution functions and their uncertainties at next-to-next-to-leading order
}

\author{
Hamzeh Khanpour ${ }^{1,2, *}$ and S. Atashbar Tehrani ${ }^{3, \dagger}$ \\ ${ }^{1}$ Department of Physics, University of Science and Technology of Mazandaran, \\ P.O.Box 48518-78195 Behshahr, Iran \\ ${ }^{2}$ School of Particles and Accelerators, Institute for Research in Fundamental Sciences (IPM), \\ P.O.Box 19395-5531 Tehran, Iran \\ ${ }^{3}$ Independent researcher, P.O.Box 1149-8834413 Tehran, Iran \\ (Received 29 August 2015; published 26 January 2016)
}

\begin{abstract}
We perform a next-to-next-to-leading order (NNLO) analysis of nuclear parton distribution functions (nPDFs) using neutral current charged-lepton $\left(\ell^{ \pm}+\right.$nucleus) deeply inelastic scattering (DIS) data and Drell-Yan (DY) cross-section ratios $\sigma_{\mathrm{DY}}^{A} / \sigma_{\mathrm{DY}}^{A^{\prime}}$ for several nuclear targets. We study in detail the parametrizations and the atomic mass (A) dependence of the nuclear PDFs at this order. The present nuclear PDFs global analysis provides us a complete set of nuclear PDFs, $f_{i}^{(A, Z)}\left(x, Q^{2}\right)$, with a full functional dependence on $x, \mathrm{~A}, \mathrm{Q}^{2}$. The uncertainties of the obtained nuclear modification factors for each parton flavour are estimated using the well-known Hessian method. The nuclear charm quark distributions are also added into the analysis. We compare the parametrization results with the available data and the results of other nuclear PDFs groups. We found our nuclear PDFs to be in reasonably good agreement with them. The estimates of errors provided by our global analysis are rather smaller than those of other groups. In general, a very good agreement is achieved. We also briefly review the recent heavy-ion collisions data including the first experimental data from the LHC proton + lead and lead + lead run which can be used in the global fits of nuclear PDFs. We highlight different aspects of the high luminosity $\mathrm{Pb}-\mathrm{Pb}$ and $\mathrm{p}-\mathrm{Pb}$ data which have been recorded by the CMS Collaboration.
\end{abstract}

DOI: $10.1103 /$ PhysRevD.93.014026

\section{INTRODUCTION}

Deep inelastic scattering (DIS) processes in HERA and hadron collisions in Tevatron and CERN-LHC provide very important tools for probing the quarks momentum distributions in the nucleons and in the nuclei. In order to describe the structure of colliding hadrons in DIS processes, a precise knowledge of the parton distribution functions (PDFs) is required. In order to achieve a better set of PDFs, many groups perform and update their global analyses of PDFs for protons [1-21] and also for nuclei [22-39]. Excellent global fits for the free proton PDFs and nuclear PDFs have been obtained by the mentioned phenomenological groups. Also a neural network techniques have been successfully developed by NNPDF group [40-43]. The accuracy of the mentioned PDFs determinations has steadily improved over the recent years, both due to more accurate DIS data and also due to improvements in perturbation theory predictions for the hard parton scattering reactions. Since the first indications that the DIS structure functions measured in the charged-lepton scattering of the nuclei, $\left(\ell^{ \pm}+\right.$nucleus $)$, differ significantly from those measured in the isolated nucleons, there has been also a continuous interest in fully understanding the

\footnotetext{
*Hamzeh.Khanpour@mail.ipm.ir

†Atashbar@ipm.ir
}

microscopic mechanism responsible in the nuclei. The importance of nuclear effects in parton distribution functions is due to the interpretation of any hard-process results involving nuclei in $\mathrm{p}+\mathrm{A}$ [44], $\mathrm{d}+\mathrm{A}$ [45] and $\mathrm{A}+\mathrm{A}$ $[46,47]$ collisions, such as heavy ions collisions at the present BNL-RHIC [48,49] and CERN-LHC [50] colliders and also future proposed electron-nucleon colliders such as EIC, eRHIC or the LHeC [51-56]. These DIS data play an important role for the observed nuclear modifications. The clean experimental environment in the DIS experiment at eRHIC and $\mathrm{LHeC}$ would provide a unique opportunity to investigate the nuclear PDFs properties. The nuclear PDFs determination in every QCD analysis have large uncertainties and are not fully constrained by the available DIS data, consequently further constraints for nuclear PDFs, especially gluons, in such high-energy nuclear colliders in the yet unexplored regions of the $x$ and $\mathrm{Q}^{2}$ plane, are most welcome.

Information about the nuclear PDFs of the nuclei can be extracted from high-energy measurements involving nuclei. Statistically, most significant data that people use in their nuclear PDFs analysis are the deep inelastic scattering (DIS) experiment which have been taken by experimental groups in fixed-target experiments. These data incorporates: The SLAC (Stanford Linear Accelerator Center)-E49, E87, E139 and E140 Collaborations, the NMC (New Muon 
Collaboration), the EMC (European Muon Collaboration), the BCDMS (Bologna-CERN-Dubna-Munich-Saclay), HERMES, JLAB groups (Jefferson Lab), the Fermilab (Fermi National Accelerator Laboratory)-E665 Collaboration, the Drell-Yan data from the Fermilab-E772 and E866/ NuSea Collaborations [57-75]. The mentioned data confirm a specific feature of the nuclear reactions called EMC effect at certain region of $x$-Bjorken. The nuclear PDFs are extracted from global analysis to a wide range of experimental data points. Owing to the complementary nature of the different DIS measurements, tight constraints on the nuclear PDFs can be obtained. A reliable extraction of nuclear PDFs from the experimental data is required for deeper understanding of the mechanism associated in hard nuclear reactions at RHIC, CERN-LHC and future electronheavy ion collision. As a result, the kinematic range of data as well as the precise determination of nuclear PDFs will continue to be a topical issue in lots of area of high energy nuclear physics program.

The main difficulties of any global analysis of nuclear PDFs are the lack of precise experimental data points that we have and fewer types of the data covering kinematical region of $x$ and $Q^{2}$ which lead to less constraints than the free proton case and also the atomic mass (A) dependence of the nuclear PDFs parameters. Consequently, the nuclear PDFs determination are not simply as the parton densities in the nucleons. In addition still more precise DIS data are needed, especially on the nuclear antiquark and gluon distributions at very low $x$ to constrain the initial state for the future RHIC and CERN-LHC programs. The DIS data of charged leptons off heavy ion targets are still used in all global nuclear PDFs analyses and provide the best constraints on nuclear modification factors for different parton distributions. These DIS data incorporate a wide range of nuclei from helium to lead which is presented as structure function ratios for different nuclei covered the range of $0.005 \lesssim x \lesssim 1$. The mentioned data will provide enough constraint in obtaining the valence quark distributions. The available data on Drell-Yan (DY) dilepton production of heavy ion target can mainly provide probes a good discrimination between valence and sea quarks distribution in the nuclei. As we mentioned, these type of data only loosely constrain the nuclear modification of gluon distribution due to the limited range in the hard energy scale. As a result, the kinematic range of data as well as the precise determination of nuclear PDFs will continue to be a topical issue in lots of areas of high energy nuclear physics program.

In the present article, we shall present for the first time, a very good quality of the nuclear PDFs using the global analysis of available experimental data, taking into account the ratio of the most commonly analyzed data sets of the structure function ratios, $F_{2}^{A} / F_{2}^{A^{\prime}}$, and Drell-Yan (DY) cross-section ratios $\sigma_{\mathrm{DY}}^{p A} / \sigma_{\mathrm{DY}}^{p A^{\prime}}$. Since the first of the nuclear PDFs sets, AT12 [22], the procedure has been improved by performing the analysis at the next-to-next-to-leading order (NNLO). An important and appealing feature of the present global QCD analysis of the nuclear PDFs is that we used the theoretical predictions at the next-to-next-to-leading order (NNLO) accuracy in perturbative QCD. We have performed a careful estimation of the uncertainties using the most common and practical method, the "Hessian method" for the nuclear modification factors of the gluons and quarks originating from the experimental errors. The resulting eigenvector sets of the nuclear PDFs can be used to propagate uncertainties to any other desired observable. The zero-mass variable flavour number scheme (ZMVFNS) is used in our analysis in order to consider the heavy quarks contributions.

The present nuclear PDFs are characterized by the full functional dependence on $x, \mathrm{Q}^{2}$ and atomic mass number (A). We also introduce the additional A dependence directly to the coefficients of the nuclear PDFs at input scale. As in other available nuclear PDFs, we also consider a flavor asymmetric antiquark distributions. We found no unusual large uncertainties for nuclear modification factor of the gluon density at medium to large $x$ obtained in some other nuclear PDFs analyses. Our global analysis considerably leads to smaller value of uncertainties in comparison with other nuclear PDFs global analyses. A detailed comparison with other available nuclear PDFs results including EPS0 9, HKN0 7, AT12, nDS and DSSZ12 have been presented. We also focus on the roles of the NNLO terms on the nuclear PDFs determination by comparing the available NLO results with our NNLO analysis. The main features of our present NNLO parametrization of nuclear PDFs are worth emphasizing already at this point. It is clear that for a precise nuclear PDFs analysis, more precise data and future advances in the theory will be needed.

The rest of the present paper consists of the following sections. In Sec. II, we shall provide a formalism to establish an analysis method and a brief discussion of the theoretical structure of the nuclear PDFs, where they arise in the calculation of DIS cross-sections and further theoretical background relevant to the reliable determination of the nuclear PDFs from experimental data. A brief summary of experimental measurements which are used in the determination of nuclear PDFs is provided in Sec. III. The analysis method and the error calculation based on the Hessian method are discussed in Sec. IV. The results of the present nuclear PDFs analysis are given in Sec. V. In Sec. VI a detailed comparison between the present results and available experimental data are presented. We have attempted a detailed comparison of our NNLO results with recent results from the literature in Sec. VII. A brief discussion on recent heavy-ion collisions including the first experimental data from the CERN-LHC proton + lead and lead + lead collisions are presented in Sec. VIII. Finally, we have presented our summary and conclusions in Sec. IX. In Appendix A, we present more details on the 
parametrization and in Appendix B a code is provided for calculating the nuclear PDFs including their uncertainties at given $x$ and $\mathrm{Q}^{2}$ in the NNLO approximation.

\section{NUCLEAR PDFS ANALYSIS METHOD}

In this section, we present our method for global analysis of nuclear PDFs (nPDFs) at next-to-next-to-leading order (NNLO). In order to calculate the parton distribution in nuclei, we need the parton distributions in a free proton. We used the following standard parameterizations at the input scale $\mathrm{Q}_{0}^{2}=2 \mathrm{GeV}^{2}$ for all parton species $x q$, obtained from JR09 set of the free proton PDFs [19],

$$
\begin{aligned}
x u_{v}\left(x, Q_{0}^{2}\right)= & 3.2350 x^{0.6710}(1-x)^{3.9293} \\
& \times\left(1-0.5302 x^{0.5}+3.9029 x\right) \\
x d_{v}\left(x, Q_{0}^{2}\right)= & 13.058 x^{1.0701}(1-x)^{6.2177} \\
& \times\left(1-2.5830 x^{0.5}+3.8965 x\right) \\
x \Delta\left(x, Q_{0}^{2}\right)= & 8.1558 x^{1.328}(1-x)^{21.043} \\
& \times\left(1-7.6334 x^{0.5}+20.054 x\right) \\
x \bar{u}+x \bar{d}= & 0.4250 x^{-0.1098}(1-x)^{10.34} \\
& \times\left(1-3.0946 x^{0.5}+11.613 x\right) \\
x g= & 3.0076 x^{0.0637}(1-x)^{5.54473} .
\end{aligned}
$$

Here $x u_{v}, x d_{v}$ represent the valance quark distributions, $x(\bar{d}+\bar{u})$ the antiquark distributions, $x \Delta=x(\bar{d}-\bar{u})$, the strange sea distribution $x s=x \bar{s}=\frac{1}{4} x(\bar{d}+\bar{u})$ and the gluon distribution, $x g$. The nuclear modifications are provided by a number of parameters at a fixed $\mathrm{Q}^{2}$ which are normally denoted by $\mathrm{Q}_{0}^{2}$. The nuclear PDFs are related to the PDFs in a free proton and for this purpose nucleonic PDFs are multiplied by a weight function $w_{i}(x, A, Z)$. With the PDFs for a bound proton inside a nucleus $\mathrm{A}, f_{i}\left(x, Q_{0}^{2}\right)$, one can reconstruct the PDFs for a general nucleus $(\mathrm{A}, \mathrm{Z})$ as follows:

$$
f_{i}^{(A, Z)}\left(x, Q_{0}^{2}\right)=w_{i}(x, A, Z) f_{i}^{\mathrm{JR} 09}\left(x, Q_{0}^{2}\right),
$$

where $f_{i}^{\mathrm{JR} 09}\left(x, Q_{0}^{2}\right)$ are coming from JR09 parametrization [19] as they were introduced by Eq. (1). Here we follow the analysis given by $[22,25,30,50,76,77]$ and assume the following functional form for the nuclear modification as a weight function,

$$
\begin{aligned}
w_{i}(x, A, Z)= & 1+\left(1-\frac{1}{A^{\alpha}}\right) \\
& \times \frac{a_{i}(A, Z)+b_{i}(A) x+c_{i}(A) x^{2}+d_{i}(A) x^{3}}{(1-x)^{\beta_{i}}} .
\end{aligned}
$$

The parameters in weight function are obtained by a global $\chi^{2}$ analysis procedure which are dependent on Bjorken variable $x$, mass number $\mathrm{A}$, and atomic number $\mathrm{Z}$. The important feature of the present analysis is that we let the free parameters of the weight function to have atomic number (A) dependencies. In order to accommodate various nuclear target materials, we introduce a nuclear A dependence in the weight function coefficients,

$b_{i}(A) \rightarrow b_{1} A^{b_{2}} ; \quad c_{i}(A) \rightarrow c_{1} A^{c_{2}} ; \quad d_{i}(A) \rightarrow d_{1} A^{d_{2}}$

$a_{\bar{q}}(A) \rightarrow a_{1} A^{a_{2}}$.

Combining the weight function in Eq. (3) with PDFs of Eq. (1), will yield us nuclear PDFs as in what follows:

$$
\begin{aligned}
& u_{v}^{(A, Z)}\left(x, Q_{0}^{2}\right)=w_{u_{v}}(x, A, Z) \frac{Z u_{v}\left(x, Q_{0}^{2}\right)+N d_{v}\left(x, Q_{0}^{2}\right)}{A}, \\
& d_{v}^{(A, Z)}\left(x, Q_{0}^{2}\right)=w_{d_{v}}(x, A, Z) \frac{Z d_{v}\left(x, Q_{0}^{2}\right)+N u_{v}\left(x, Q_{0}^{2}\right)}{A}, \\
& \bar{u}^{(A, Z)}\left(x, Q_{0}^{2}\right)=w_{\bar{q}}(x, A, Z) \frac{Z \bar{u}\left(x, Q_{0}^{2}\right)+N \bar{d}\left(x, Q_{0}^{2}\right)}{A}, \\
& \bar{d}^{(A, Z)}\left(x, Q_{0}^{2}\right)=w_{\bar{q}}(x, A, Z) \frac{Z \bar{d}\left(x, Q_{0}^{2}\right)+N \bar{u}\left(x, Q_{0}^{2}\right)}{A}, \\
& s^{(A, Z)}\left(x, Q_{0}^{2}\right)=\bar{s}^{(A, Z)}\left(x, Q_{0}^{2}\right)=w_{\bar{q}}(x, A, Z) s\left(x, Q_{0}^{2}\right), \\
& g^{(A, Z)}\left(x, Q_{0}^{2}\right)=w_{g}(x, A, Z) g\left(x, Q_{0}^{2}\right) .
\end{aligned}
$$

In the first four equations, the $\mathrm{Z}$ term as atomic number indicates the number of protons and the $\mathrm{N}=\mathrm{A}-\mathrm{Z}$ term represents the number of neutrons in the nuclei while the $\mathrm{SU}(3)$ symmetry is apparently broken there. We find that the parametrization in Eq. (5) is sufficiently flexible to allow a good $\chi^{2}$ fit to the available data sets.

If the number of protons and neutrons in a nuclei are equal to each other (isoscalar nuclei) such as ${ }^{2} \mathrm{D},{ }^{4} \mathrm{He}$, ${ }^{12} \mathrm{C}$, and ${ }^{40} \mathrm{Ca}$ nuclei, the valence quarks $u_{v}^{(A, Z)}, d_{v}^{(A, Z)}$, $\bar{u}^{(A, Z)}$, and $\bar{d}^{(A, Z)}$ would have similar distributions. In the case that $\mathrm{Z}$ and $\mathrm{A}$ numbers are not equal in the nuclei, it can be concluded that antiquark distributions $\bar{u}^{(A, Z)}$, $\bar{d}^{(A, Z)}$, and $\bar{s}^{(A, Z)}$ in the nuclei would not be equal to each other $[22,78,79]$. For the strange quark distributions in the nuclei some research studies are still being done [80] but we assume the common case in which it is assumed $(s=\bar{s})$.

In Eq. (3) we fixed $\alpha=\frac{1}{3}$, considering the constraints which are imposed by nuclear volume and surface contributions. The parameters $b_{i}(A), c_{i}(A)$, and $d_{i}(A)$ which are listed in Eq. (4), will be directly determined from the global $\chi^{2}$ fits. The Fermi motion part parameter $\beta_{i}$ cannot be determined from fit due to the lack of experimental data. We fixed them to $\beta_{v}=0.4, \beta_{\bar{q}}=0.1$, and $\beta_{g}=0.1$ for valence, sea quark, and gluon distributions respectively. 
There are three constraints for the parameters namely the nuclear charge $\mathrm{Z}$, baryon number (mass number) $\mathrm{A}$ and momentum conservations $[22,28,29,81]$,

$$
\begin{aligned}
Z= & \int \frac{A}{3}\left[2 u_{v}^{A}-d_{v}^{A}\right]\left(x, Q_{0}^{2}\right) d x, \\
3= & \int\left[u_{v}^{A}+d_{v}^{A}\right]\left(x, Q_{0}^{2}\right) d x, \\
1= & \int x\left[u_{v}^{A}+d_{v}^{A}+2\left\{\bar{u}^{A}+\bar{d}^{A}+\bar{s}^{A}\right\}\right. \\
& \left.+g^{A}\right]\left(x, Q_{0}^{2}\right) d x .
\end{aligned}
$$

The $a_{i}(A, Z)$ parameters for the $u_{v}$ and $d_{v}$ distributions $\left(a_{v}\right)$ are fixed by the nuclear charge $\mathrm{Z}$ and baryon number $\mathrm{A}$ conservations, while $a_{g}$ parameters for the gluon distribution, is fixed by the existing momentum sum rule in Eq. (6).

In our calculations, we take $\mathrm{Q}_{0}^{2}=2 \mathrm{GeV}^{2}$ and the $\chi^{2}$ analysis is done based on the well-known DGLAP evolution equations [82]. Our calculations are done at the nextnext-to-leading (NNLO) approximation in which the modified minimal subtraction scheme $(\overline{M S})$ is used. In our previous next-to-leading order nuclear PDFs analysis [22], the NLO version of the KKT PDFs fit was employed $[3,4]$. Since the NNLO version of the mention PDFs fits are not available yet, the JR09 (Jimenez-Delgado, Reya) nucleonic PDFs parametrization [19] is used in the present analysis. According to their analysis, the strange quark PDF is assumed to be symmetric $(x s=x \bar{s})$ and it is proportional to the isoscalar light quark sea and parametrized as

$$
x s=x \bar{s}=k(\bar{d}+\bar{u}),
$$

with $k=\frac{1}{4}$. In Fig. 1, we plot the NNLO parton distribution functions of JR09 at the input scale $\mathrm{Q}_{0}^{2}=2 \mathrm{GeV}^{2}$.

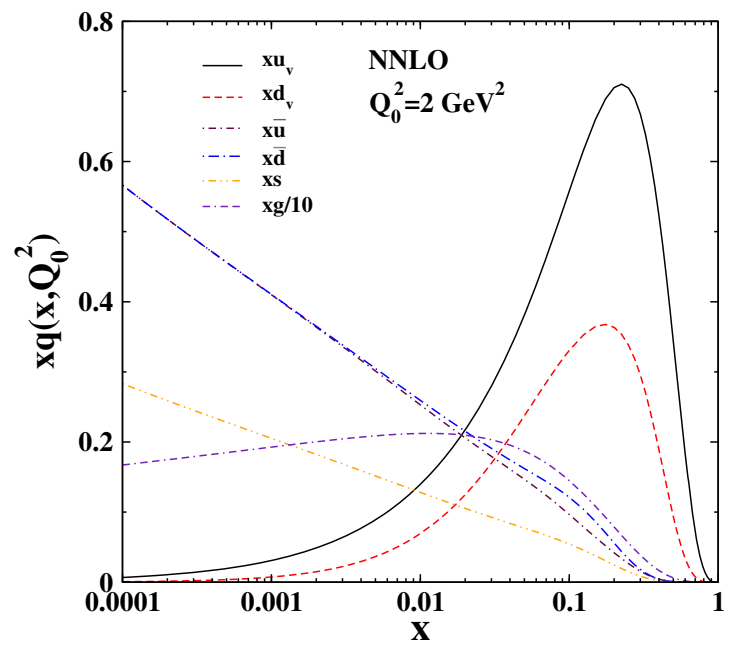

FIG. 1. The input PDFs from JR09 [19] at the input scale $Q_{0}^{2}=$ $2 \mathrm{GeV}^{2}$ at NNLO approximation.
For the $\mathrm{Q}^{2}$ evolution and in order to account for the heavy quarks contributions, we choose the zero-mass variable flavor number scheme (ZM-VFNS) with the charm flavor threshold set at $m_{c}=1.40 \mathrm{GeV}$. We add the nuclear charm quark distributions into the present nuclear PDFs analysis. In the ZM-VFNS, the only explicit dependance on the quark masses in the value at which the number of active flavors changes. We let the heavy quarks to be massless and generate them through the DGLAP evolution above the mass thresholds.

The $F_{2}^{(A, Z)}\left(x, Q^{2}\right)$ structure functions can be extracted at NNLO approximation as a convolutions of nuclear PDFs of Eq. (2) with the corresponding Wilson coefficients [83-85],

$$
F_{2}^{(A, Z)}\left(x, Q^{2}\right)=\sum_{i=u, d, s, g} C_{i} \otimes f_{i}^{(A, Z)}\left(x, Q^{2}\right) .
$$

Consequently the nuclear structure functions are given by

$$
\begin{aligned}
F_{2}^{(A, Z)}\left(x, Q^{2}\right)= & \sum_{i=u, d, s} e_{i}^{2} x\left[1+a_{s} C_{q}^{1}(x)+a_{s}^{2} C_{q}^{2}(x)\right] \\
& \otimes\left(q_{i}^{A}+\bar{q}_{i}^{A}\right) . \\
& +\frac{1}{2 f}\left(a_{s} C_{g}^{1}(x)+a_{s}^{2} C_{g}^{2}(x)\right) \otimes x g .
\end{aligned}
$$

In this equation, $C_{q, g}^{1,2}$ are the common Wilson coefficient at NLO and NNLO approximation $[84,85]$ and the symbol $\otimes$ denotes the usual convolution integral,

$$
f(x) \otimes g(x)=\int_{x}^{1} \frac{\mathrm{DY}}{y} f\left(\frac{x}{y}\right) g(y) .
$$

\section{INPUT TO THE GLOBAL NUCLEAR PDFS FIT}

In the present section, we review the available experimental data including charged-lepton $\left(\ell^{ \pm}+\right.$nucleus) DIS and Drell-Yan cross-section ratios $\sigma_{\mathrm{DY}}^{p A} / \sigma_{\mathrm{DY}}^{p A^{\prime}}$ for different nuclear targets as the input for the global fit. In order to include the heavy-target data into a global analysis of proton PDFs, the nuclear corrections are considered. Using these variety of $\ell^{ \pm} \mathrm{A}$ and Drell-Yan data, we can construct global nuclear PDFs fit. A large and complete experimental data sets for different nuclear targets in wide range of $x$ and $\mathrm{Q}^{2}$ required to fully constraints the $x, \mathrm{~A}, \mathrm{Q}^{2}$ and also for flavour dependencies of the nuclear PDFs. The nuclear effects have been studied experimentally in charged leptonnucleus scattering by some experimental groups such as the muon experiments BCDMS, EMC, and NMC at CERN, EMC-NA38 and E665 at FNAL, in electron scattering at SLAC, DESY, and JLAB, in the Drell-Yan process and also in neutrino-nucleus scattering.

The $x$ and $\mathrm{Q}^{2}$ coverage of the data sets used in our nuclear PDFs fits are illustrated in Fig. 2. The interval range of the $\mathrm{Q}^{2}$ values is $\mathrm{Q}^{2} \geq 1 \mathrm{GeV}^{2}$ and the smallest value for 


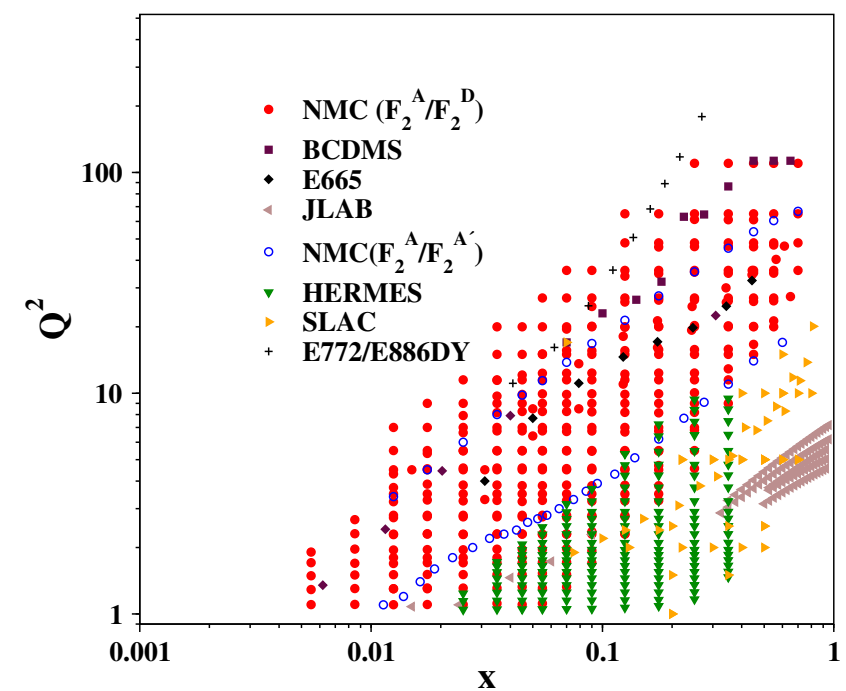

FIG. 2. Nominal coverage of the data sets used in our global fits. The plot nicely summarizes the universal $x$ dependance of the nuclear effect.

the $x$-Bjorken variable is equal to 0.0055 at this stage. Nominally there is a substantial amount of data at larger Bjorken variable $x$. The plot clearly shows the worse coverage of the data at medium to small- $x$ region.

As Fig. 2 clearly shows, these data sets are later limited in comparison with the data for free proton PDFs. The proton fit uses a very large and very precise data from Tevatron and HERA colliders while the nuclear PDFs uses a smaller data sample from several fixed target experiments and some collider data from RHIC. Consequently, lacking precision and smaller amount of the nuclear data specially at small value of $x$-Bjorken, could lead to larger uncertainties for nuclear PDFs than the PDFs for the free proton. Consequently for better and precise determination of nuclear quark and gluon distributions, especially for very low parton momentum fractions $x$, further measurements for the EMC effect in the neutrino-nucleus, electronnucleus and proton-nucleus scattering are needed.

The total experimental data sets that we used in our present global analysis are listed in Table I. The $F_{2}^{A}\left(F_{2}^{A^{\prime}}\right)$ is

TABLE I. The charged-lepton DIS experimental data sets for $\mathrm{F}_{2}^{A} / \mathrm{F}_{2}^{D}, \mathrm{~F}_{2}^{A} / \mathrm{F}_{2}^{A^{\prime}}$ and Drell-Yan cross section ratios $\sigma_{\mathrm{DY}}^{A} / \sigma_{\mathrm{DY}}^{A^{\prime}}$ used in the present global fit. Number of data points, the related references and specific nuclear targets are also listed.

\begin{tabular}{|c|c|c|c|}
\hline Nucleus & Experiment & Number of data points & Reference \\
\hline \multicolumn{4}{|l|}{$\mathrm{F}_{2}^{A} / \mathrm{F}_{2}^{D}$} \\
\hline \multirow[t]{2}{*}{$\mathrm{He} / \mathrm{D}$} & SLAC-E139 & 18 & [69] \\
\hline & NMC-95 & 17 & [57] \\
\hline $\mathrm{Li} / \mathrm{D}$ & NMC-95 & 17 & [57] \\
\hline $\mathrm{Li} / \mathrm{D}\left(\mathrm{Q}^{2} \mathrm{dep}\right)$ & NMC-95 & 179 & [68] \\
\hline
\end{tabular}

(Table continued)
TABLE I. (Continued)

\begin{tabular}{|c|c|c|c|}
\hline Nucleus & Experiment & Number of data points & Reference \\
\hline $\mathrm{Be} / \mathrm{D}$ & SLAC-E139 & 17 & {$[58]$} \\
\hline \multirow[t]{6}{*}{$\mathrm{C} / \mathrm{D}$} & EMC-88 & 9 & [64] \\
\hline & EMC-90 & 5 & [69] \\
\hline & SLAC-E139 & 7 & {$[58]$} \\
\hline & NMC-95 & 17 & {$[57]$} \\
\hline & FNAL-E665 & 5 & [66] \\
\hline & JLAB-E03-103 & 103 & [67] \\
\hline $\mathrm{C} / \mathrm{D}\left(\mathrm{Q}^{2} \mathrm{dep}\right)$ & NMC-95 & 191 & [68] \\
\hline \multirow[t]{2}{*}{$\mathrm{N} / \mathrm{D}$} & BCDMS-85 & 9 & [70] \\
\hline & HERMES-03 & 153 & [73] \\
\hline \multirow[t]{2}{*}{$\mathrm{Al} / \mathrm{D}$} & SLAC-E49 & 18 & [59] \\
\hline & SLAC-E139 & 17 & {$[58]$} \\
\hline \multirow[t]{4}{*}{$\mathrm{Ca} / \mathrm{D}$} & EMC-90 & 5 & [69] \\
\hline & NMC-95 & 16 & {$[57]$} \\
\hline & SLAC-E139 & 7 & [69] \\
\hline & FNAL-E665 & 5 & {$[66]$} \\
\hline \multirow[t]{4}{*}{$\mathrm{Fe} / \mathrm{D}$} & SLAC-E87 & 14 & {$[60]$} \\
\hline & SLAC-E139 & 23 & {$[58]$} \\
\hline & SLAC-E140 & 10 & [61] \\
\hline & BCDMS-87 & 10 & [62] \\
\hline $\mathrm{Cu} / \mathrm{D}$ & EMC-93 & 19 & [63] \\
\hline $\mathrm{Kr} / \mathrm{D}$ & HERMES-03 & 144 & [73] \\
\hline $\mathrm{Ag} / \mathrm{D}$ & SLAC-E139 & 7 & [58] \\
\hline $\mathrm{Sn} / \mathrm{D}$ & EMC-88 & 8 & [64] \\
\hline $\mathrm{Xe} / \mathrm{D}$ & FNAL-E665-92 & 5 & [65] \\
\hline \multirow[t]{2}{*}{$\mathrm{Au} / \mathrm{D}$} & SLAC-E139 & 18 & {$[58]$} \\
\hline & SLAC-E140 & 1 & [61] \\
\hline $\mathrm{Pb} / \mathrm{D}$ & FNAL-E665-95 & 5 & [66] \\
\hline \multicolumn{4}{|l|}{$\mathrm{F}_{2}^{A} / \mathrm{F}_{2}^{A^{\prime}}$} \\
\hline $\mathrm{Be} / \mathrm{C}$ & NMC-96 & 15 & [71] \\
\hline $\mathrm{Al} / \mathrm{C}$ & NMC-96 & 15 & [71] \\
\hline \multirow[t]{2}{*}{$\mathrm{Ca} / \mathrm{C}$} & NMC-96 & 24 & [57] \\
\hline & NMC-96 & 15 & [71] \\
\hline $\mathrm{Fe} / \mathrm{C}$ & NMC-96 & 15 & [71] \\
\hline \multirow[t]{2}{*}{$\mathrm{Sn} / \mathrm{C}$} & NMC-96 & 146 & [71] \\
\hline & NMC-96 & 15 & [72] \\
\hline $\mathrm{Pb} / \mathrm{C}$ & NMC-96 & 15 & [71] \\
\hline $\mathrm{C} / \mathrm{Li}$ & NMC-95 & 24 & [57] \\
\hline $\mathrm{Ca} / \mathrm{Li}$ & NMC-95 & 24 & [57] \\
\hline \multicolumn{4}{|l|}{$\sigma_{\mathrm{DY}}^{A} / \sigma_{\mathrm{DY}}^{A^{\prime}}$} \\
\hline $\mathrm{Fe} / \mathrm{Be}$ & FNAL-E866/] & NuSea & [74] \\
\hline $\mathrm{W} / \mathrm{Be}$ & FNAL-E866/] & NuSea & [74] \\
\hline $\mathrm{C} / \mathrm{D}$ & FNAL-E77 & $2-90$ & [75] \\
\hline $\mathrm{Ca} / \mathrm{D}$ & FNAL-E77 & $2-90$ & [75] \\
\hline $\mathrm{Fe} / \mathrm{D}$ & FNAL-E77 & $2-90$ & [75] \\
\hline $\mathrm{W} / \mathrm{D}$ & FNAL-E77 & $2-90$ & [75] \\
\hline Total & & 1479 & \\
\hline
\end{tabular}

denoting the structure function of a nuclei and $F_{2}^{D}$ is representing the structure function of deuterium. Number of data points, together with the related references and specific nuclear targets are also listed in the table. The total 
number of data sets for the $F_{2}^{A} / F_{2}^{D}$ ratios are equal to 1079 and the number of $F_{2}^{A} / F_{2}^{A^{\prime}}$ ratio for $\mathrm{Be} / \mathrm{C}, \mathrm{Al} / \mathrm{C}$, $\mathrm{Ca} / \mathrm{C}, \mathrm{Fe} / \mathrm{C}, \mathrm{Sn} / \mathrm{C}, \mathrm{Pb} / \mathrm{C}, \mathrm{C} / \mathrm{Li}$ is 308 . The data comes from the Drell-Yan process provide a complementary constraint on the nuclear PDFs. In particular, they allow one to separate the sea quark distributions in the nuclei. For this purpose, we use the data obtained by FNALE866 [74] and FNAL-E772 [75] experiments at Fermilab. For the Drell-Yan cross section ratios $\left(\sigma_{\mathrm{DY}}^{p A} / \sigma_{\mathrm{DY}}^{p A^{\prime}}\right)$ we have 92 data points while the related ratio are $C / D$, $\mathrm{Ca} / \mathrm{D}, \mathrm{Fe} / \mathrm{D}, \mathrm{W} / \mathrm{D}, \mathrm{Fe} / \mathrm{Be}$, and $\mathrm{W} / \mathrm{Be}$. The total experimental data points were included in our analysis is 1479. They contain lepton-nucleus deep inelastic scattering $\left(\ell^{ \pm}+\right.$nucleus $)$and Drell-Yan cross-section ratios $\sigma_{\mathrm{DY}}^{p A} / \sigma_{\mathrm{DY}}^{p A^{\prime}}$ data for different nuclear targets.

\section{THE ANALYSIS OF $\chi^{2}$ VALUE AND ERROR CALCULATION VIA HESSIAN METHOD}

To determine the best fit at NNLO, we need to minimize the $\chi^{2}$ with respect to 16 free input nuclear PDFs parameters of Eq. (5). The global goodness-of-fit procedure follows the usual chi-squared method with $\chi^{2}(p)$ defined as

$$
\chi^{2}(p)=\sum_{i=1}^{n^{\text {data }}} \frac{\left(R_{i}^{\text {data }}-R_{i}^{\text {theory }}(p)\right)^{2}}{\left(\sigma_{i}^{\text {data }}\right)^{2}},
$$

where $p$ denotes the set of 16 independent parameters in the fit and $n^{\text {data }}$ is the number of data points included, $n^{\text {data }}=1479$ for the NNLO fit. The optimization of the above $\chi^{2}$ value to determine the best parametrization of the nuclear PDFs is done by the CERN program library MINUIT [86]. For the $i$ th experiment, $R_{i}^{\text {data }}, \sigma_{i}^{\text {data }}$, and $R_{i}^{\text {theory }}$ denote the experimental data value, measured uncertainty and theoretical value for the $n$th data point. The experimental errors are calculated from systematic and statistical errors, $\sigma_{i}^{\text {data }}=\sqrt{\left(\sigma_{i}^{\text {sys }}\right)^{2}+\left(\sigma_{i}^{\text {stat }}\right)^{2}}$. The theory prediction $R_{i}^{\text {theory }}$, which is denoting the theoretical result of $F_{2}^{p A} / F_{2}^{p A^{\prime}}$ and $\sigma_{\mathrm{DY}}^{p A} / \sigma_{\mathrm{DY}}^{p A^{\prime}}$ ratios, depends on the input nuclear PDFs parameters $p$.

For the error calculation, a standard error analysis is needed for the nuclear PDFs by taking into account correlations among the parameters. The method to consider the correlations among the uncertainties are discussed in detail in Refs. [12,22,87-90], so we explain only a brief outline here. Following that, an error analysis can be done using the Hessian or covariance matrix, which is obtained by running the CERN program library MINUIT. The nuclear PDFs uncertainties are estimated, using the Hessian matrix as the following

$$
\begin{aligned}
& \delta f^{A}(x) \\
& =\left[\Delta \chi^{2} \sum_{i, j}\left(\frac{\partial f^{A}(x, \xi)}{\partial \xi_{i}}\right)_{\xi=\hat{\xi}} H_{i j}^{-1}\left(\frac{\partial f^{A}(x, \xi)}{\partial \xi_{j}}\right)_{\xi=\hat{\xi}}\right]^{1 / 2},
\end{aligned}
$$

where the $H_{i j}$ is the Hessian matrix (also known as the error matrix), $\xi_{i}$ is the quantity referring to the parameters which exist in nuclear PDFs and $\hat{\xi}$ indicates the number of parameters which make an extremum value for the related derivative. We are able to calculate the nuclear PDFs uncertainties using these covariance matrix elements based on the method as mentioned in this section. Their values at higher $\mathrm{Q}^{2}>\mathrm{Q}_{0}^{2}$ are calculated by the well-known DGLAP evolution equations.

The well-known Hessian method which is based on the covariance matrix diagonalization, provides us a simple and efficient method for calculating the PDFs uncertainty $[12,22,88-90]$. In this method, one can assume that the deviation in the global goodness-of-fit quantity, $\Delta \chi_{\text {global }}^{2}$, is quadratic in the deviation of the parameters specifying the input parton distributions, $p_{i}$, from their values at the minimum, $p_{i}^{\mathrm{min}}$. So one can write

$$
\Delta \chi_{\text {global }}^{2} \equiv \chi_{\text {global }}^{2}-\chi_{\text {min }}^{2}=\sum_{i, j} H_{i j}\left(p_{i}-p_{i}^{\min }\right)\left(p_{j}-p_{j}^{\min }\right),
$$

where $H_{i, j}$ is an element of the Hessian matrix determined in the global nuclear PDFs fit. By having a set of appropriate nuclear PDFs fit parameters which minimize the global $\chi^{2}$ function, $s^{\mathrm{min}}$, and introducing nuclear parton sets $s_{k}^{ \pm}$, one can write

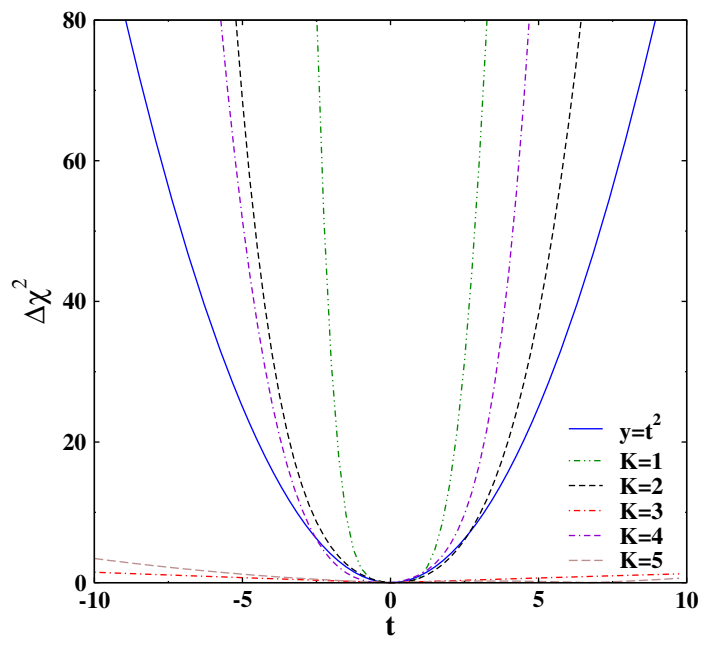

FIG. 3. $\Delta \chi^{2}$ as a function of $t$ defined in Eq. (14) for some random sample of eigenvectors. 
TABLE II. The input nuclear PDFs parameters of valance quark, sea quark and gluon distributions at $\mathrm{Q}_{0}^{2}=2 \mathrm{GeV}^{2}$ obtained by global $\chi^{2}$ analysis. The details of the $\chi^{2}$ analysis and constraints applied to control the parameters are contained in the text.

\begin{tabular}{lll}
\hline \hline$a_{v}$ & $a_{\bar{q}}(A)$ & $a_{g}$ \\
Appendix A & $-0.14364 \pm 8.938466 \times 10^{-3} A^{0.149757 \pm 1.3456148 \times 10^{-2}}$ & Appendix A \\
$b_{v}(A)$ & $b_{\bar{q}}(A)$ & $b_{g}$ \\
$1.98347 \pm 0.1705875 A^{-0.0791784 \pm 1.19181 \times 10^{-2}}$ & $3.1188 \pm 0.2080143 A^{0.159521 \pm 1.4907795 \times 10^{-2}}$ & $0.105397 \pm 2.139654$ \\
$c_{v}(A)$ & $c_{\bar{q}}(A)$ & $c_{g}$ \\
$-6.46451 \pm 0.3582447 A^{-0.038812 \pm 1.36899 \times 10^{-2}}$ & $-15.5991 \pm 1.1211789 A^{0.183694 \pm 1.8131510 \times 10^{-2}}$ & 0 \\
$d_{v}(A)$ & $d_{\bar{q}}(A)$ & $d_{g}$ \\
$4.90165 \pm 0.3045687 A^{0.00900608 \pm 1.81409 \times 10^{-2}}$ & $18.7266 \pm 2.2757606 A^{0.255328 \pm 2.9314540 \times 10^{-2}}$ & $1.48382 \pm 1.353835$ \\
$\beta_{v}$ & $\beta_{\bar{q}}$ & $\beta_{g}$ \\
0.4 Fixed & 0.1 Fixed & 0.1 Fixed \\
\hline \hline
\end{tabular}

$$
p_{i}\left(s_{k}^{ \pm}\right)=p_{i}\left(s^{\min }\right) \pm t \sqrt{\lambda_{k}} v_{i k},
$$

where $v_{i k}$ is the eigenvector and $\lambda_{k}$ is the $k^{\text {th }}$ eigenvalue. The parameter $t$ is adjusted to make the required $T=$ $\sqrt{\Delta \chi_{\text {global }}^{2}}$ global which is the allowed deterioration in $\Delta \chi_{\text {global }}^{2}$ global quality for the error determination and $t=T$ is the ideal quadratic behavior. To test the quadratic approximation of Eq. (13), we study the dependence of $\Delta \chi_{\text {global }}^{2}$ along some random samples of eigenvector directions. The $\Delta \chi_{\text {global }}^{2}$ treatment for some selected eigenvectors numbered $k=1,2,3,4$ and 5 for the presented nuclear PDFs analysis are illustrated in Fig. 3.

\section{RESULTS OF THE NUCLEAR PDFS FITS}

We are now in a position to present the results of our nuclear PDFs analysis which we call the KA15 nPDFs fit. In the following section, the results of the present nuclear PDFs studies are discussed in detail and compared with the available experimental data. In the present analysis which has been done at the NNLO approximation, we obtain an overall $\frac{\chi^{2}}{\text { d.o.f }}=1696.65 / 1463=1.15$. The total number of the data points for the nuclear structure functions and DrellYan ratios is 1479. As we mentioned, the number of parameters which is used in our fitting procedure is equal to 16 . The output of the global fit is the set of $b_{i}, c_{i}$ and $d_{i}$ parameters which are corresponding to $b(A), c(A)$, and $d(A)$. Their A-dependent functions will lead to the determination of the nuclear PDFs at the initial scale $\mathrm{Q}_{0}^{2}=2 \mathrm{GeV}^{2}, f_{i}\left(x, Q_{0}^{2}\right)$. At the first step, 20 parameters have been optimized by minimizing the usual chi-squared method $\chi^{2}(p)$ and in the second step since we have fixed four parameters $\beta_{v}=0.4, \beta_{\bar{q}}=0.1, \beta_{g}=0.1$ and $\alpha=\frac{1}{3}$, we just need to determine 16 parameters of the weight functions via our fitting procedure. In order to control the Fermi motions of the partons inside the nuclei at the larger values of $x$, we have to fix $\beta_{v}, \beta_{\bar{q}}$ and $\beta_{g}$ parameters. These parameters cannot be well determined from fit due to the lack of the experimental data. Consequently fixing these data may lead to reach a well converging (well constrained) global nuclear PDFs fit. For the nuclear modification of the valance and sea quark distributions, we choose an $A$ dependent functional form while the weight function for the gluon distribution is assumed to be independent of the A number. The numerical values of the parameters defining the modifications as well as the fixed parameters are listed in Table II. The parameters $a_{u_{v}}, a_{d_{v}}$, and $a_{g}$ are fixed by the three sum rules, given by Eq. (6) (See Appendix A of Ref. [22] for more details.). The parameter errors quoted in the table, are due to the propagation of the systematic and statistical errors in the used DIS data.

Nuclear modifications $W_{i}\left(i=u_{v}, d_{v}, \bar{q}\right.$ and $\left.g\right)$ for all the analyzed nuclei at the input scale $\mathrm{Q}_{0}^{2}=2 \mathrm{GeV}^{2}$ have been represented in Fig. 4.

As a typical heavy-sized nucleus, gold nuclei has been selected for showing the nuclear modifications in Fig. 5 and
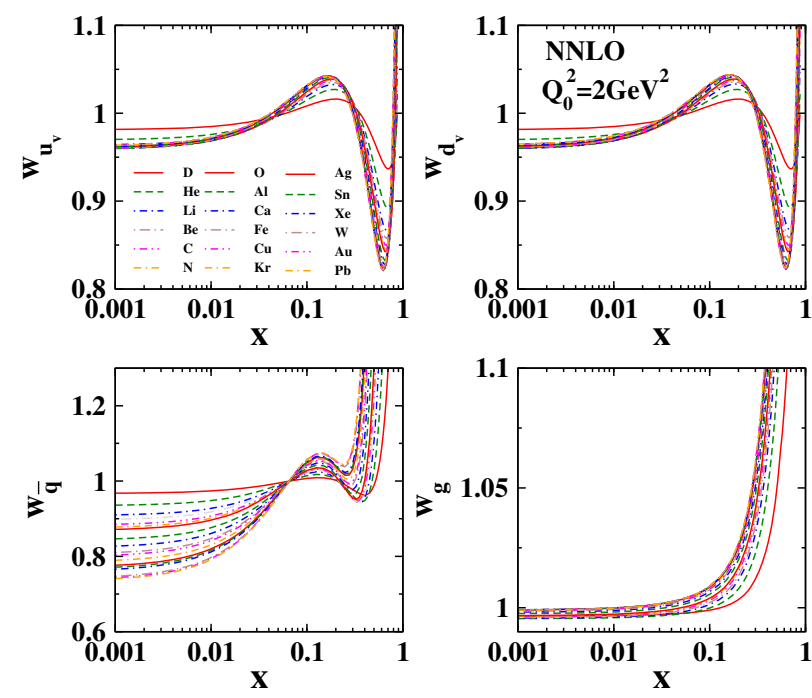

FIG. 4. Nuclear modification factors for $W_{u_{v}}, W_{d_{v}}, W_{\bar{q}}$ and $W_{g}$ are shown in the NNLO for all the analyzed nuclei at $Q_{0}^{2}=2 \mathrm{GeV}^{2}$. The nuclear mass number becomes larger in the order of $\mathrm{D}, \mathrm{He}, \mathrm{Li}, \mathrm{Be}, \ldots$, and $\mathrm{Pb}$. 

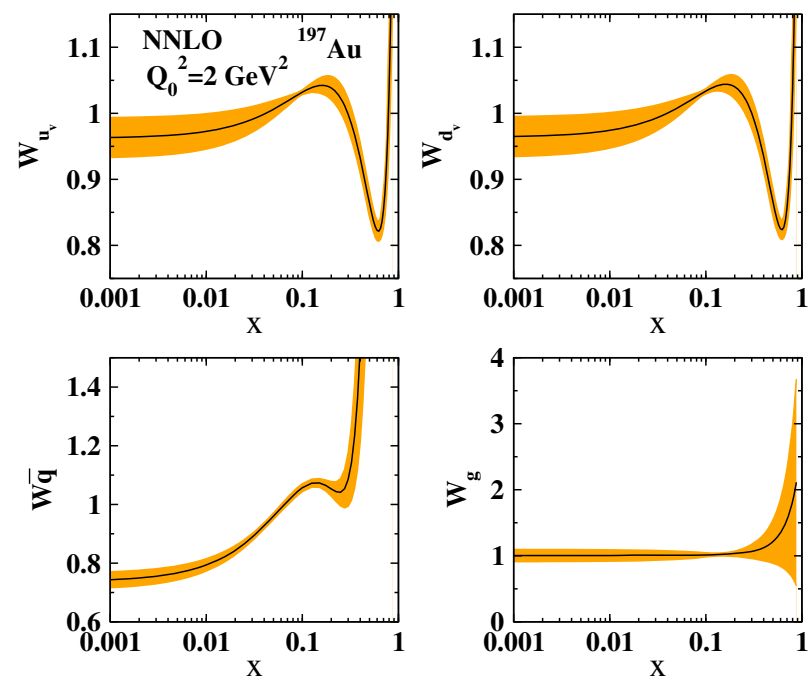

FIG. 5. Nuclear modification factors of the PDFs and their uncertainties are shown for the gold nucleus at $\mathrm{Q}_{0}^{2}=2 \mathrm{GeV}^{2}$.

the nuclear PDFs including their uncertainties in Fig. 6 at the input scale $\mathrm{Q}_{0}^{2}=2 \mathrm{GeV}^{2}$.

It is worth noting that although the nuclear PDFs for $x \bar{d}$ and $x \bar{u}$ are similar, there is small difference with $x \bar{d}>x \bar{u}$. This maybe explained by a relative suppression of the $g \rightarrow$ $u \bar{u}$ process due to the exclusion principle and the larger number of up quark which already occupied. The gluon modification and its distribution which plotted for Gold nucleus in Fig. 6 clearly show that we have a large uncertainty band at small value of the Bjorken- $x$ value.

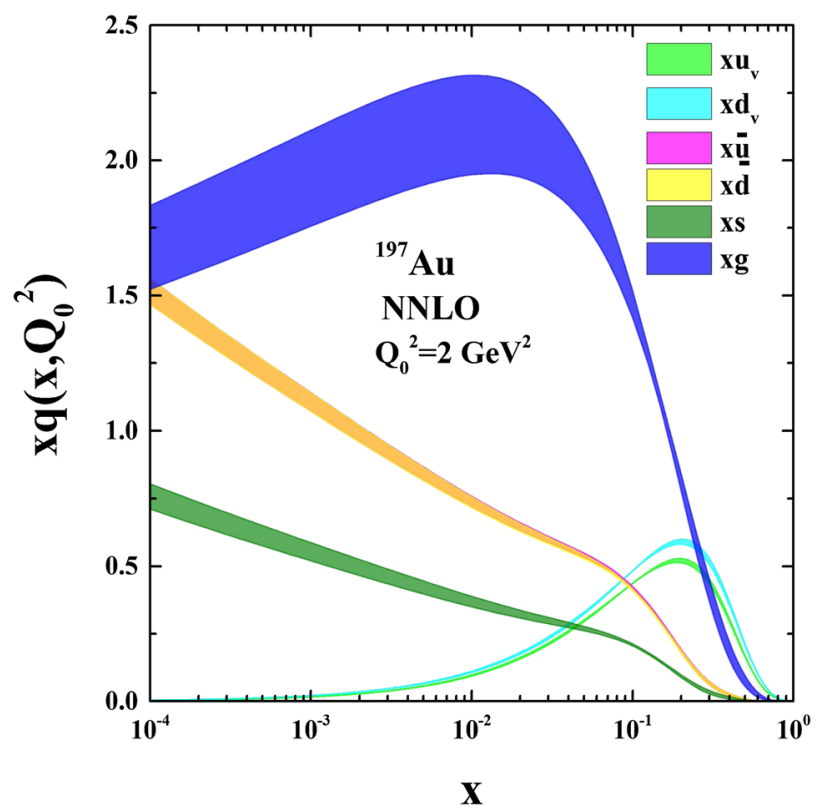

FIG. 6. The nuclear parton distribution functions in gold nucleus at $\mathrm{Q}_{0}^{2}=2 \mathrm{GeV}^{2}$ including their uncertainties. The gluon has a large uncertainty at small- $x$.
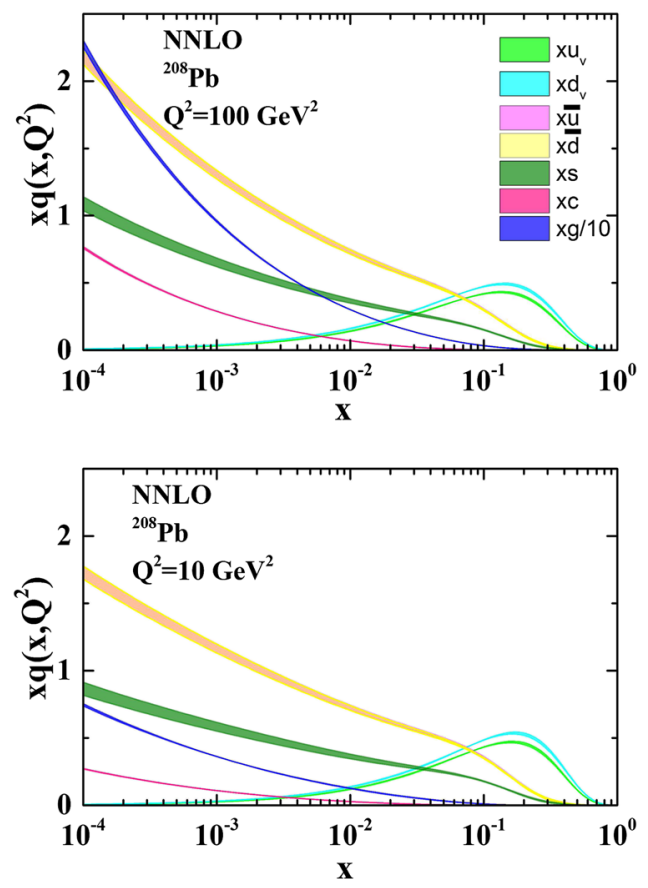

FIG. 7. Nuclear parton distribution functions in Lead at $\mathrm{Q}^{2}=$ $10 \mathrm{GeV}^{2}$ and $100 \mathrm{GeV}^{2}$ at the NNLO approximation.

The wide uncertainty band for the gluon reflects the fact that there are not enough data constraints.

Using the DGLAP evolution equations, we can evolve the $f_{i}\left(x, Q_{0}^{2}\right)$ to an arbitrary $\mathrm{Q}^{2}$ to obtain the desired nuclear PDFs $f_{i}\left(x, Q^{2}\right)$. In Figs. 7 and 8 , we display the nuclear
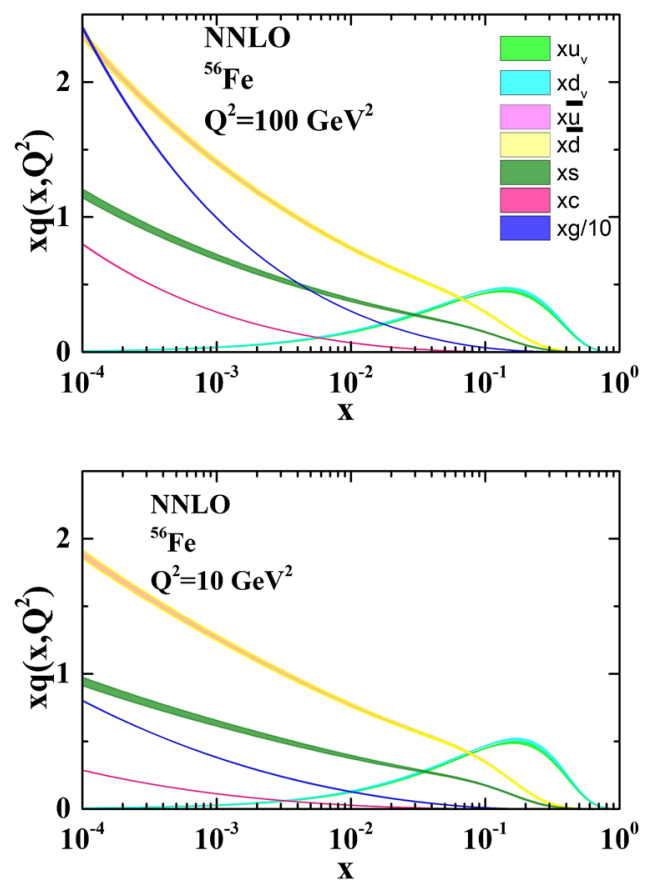

FIG. 8. Nuclear parton distribution functions in Iron at $Q^{2}=$ $10 \mathrm{GeV}^{2}$ and $100 \mathrm{GeV}^{2}$ at the NNLO approximation. 
PDFs at the $\mathrm{Q}^{2}=10 \mathrm{GeV}^{2}$ and $100 \mathrm{GeV}^{2}$ as a function of $x$ for lead and iron respectively.

The $x u_{v}, x d_{v}$ valance quark distributions, the antiquark distributions $x \bar{d}$ and $x \bar{u}$, the strange sea distribution $x s$ and also charm distribution $x c$ and the gluon distribution, $x g$ are shown as well. As the results clearly show, there are still large uncertainties in the nuclear PDFs, especially for the gluon sectors. To resolve the gluon uncertainties in nuclei at small- $x, \quad(x<0.001)$, much accurate hard scattering data from electron-A collider would be needed. Further DIS data from RHIC $d+\mathrm{Au}$ and CERN-LHC proton lead collisions, will help in constraining the nuclear PDFs. As we defined in Eq. (5), we assumed flavor asymmetric antiquark distributions, $\bar{d}^{A} \neq \bar{u}^{A}$. In the isoscalar nuclei such as ${ }^{2} \mathrm{D},{ }^{4} \mathrm{He},{ }^{12} \mathrm{C}$ and ${ }^{40} \mathrm{Ca}$, the $\bar{u}^{A}$ and $\bar{d}$ distributions are equal so we have flavor symmetry. For other nuclei which the number of their protons and neutrons are not equal, we have the $S U(3)$ flavor symmetry breaking.

Figure 9 shows a very interesting result. In this figure, the $\left(\bar{u}^{A}-\bar{d}^{A}\right) /\left(u^{A}+\bar{u}^{A}-d^{A}-\bar{d}^{A}\right)$ ratio has been shown at $\mathrm{Q}^{2}=100 \mathrm{GeV}^{2}$ for some nonisoscalar nuclei in which we have $\mathrm{SU}(3)$ symmetry breaking. The $\bar{d}^{A} \neq \bar{u}^{A}$ asymmetry are clearly shown at small $x,(x<0.05)$. This effect may be due to the sensitivity of the Drell-Yan data to the isospin asymmetry of the sea quark distributions.

In Fig. 10, we compare nuclear parton distributions of the $\mathrm{Li}, \mathrm{Al}$, and $\mathrm{Xe}$ nucleus at the $\mathrm{Q}^{2}=10 \mathrm{GeV}^{2}$ to investigate the A-dependence of the various nuclear PDFs flavours. As the plot shows, when examining the A-dependence of nuclear PDFs, we notice that the smaller nuclei has a larger value of sea-quark and gluon distributions at small value of $x$. The $\bar{d}$ and $\bar{u}$ PDFs are very similar

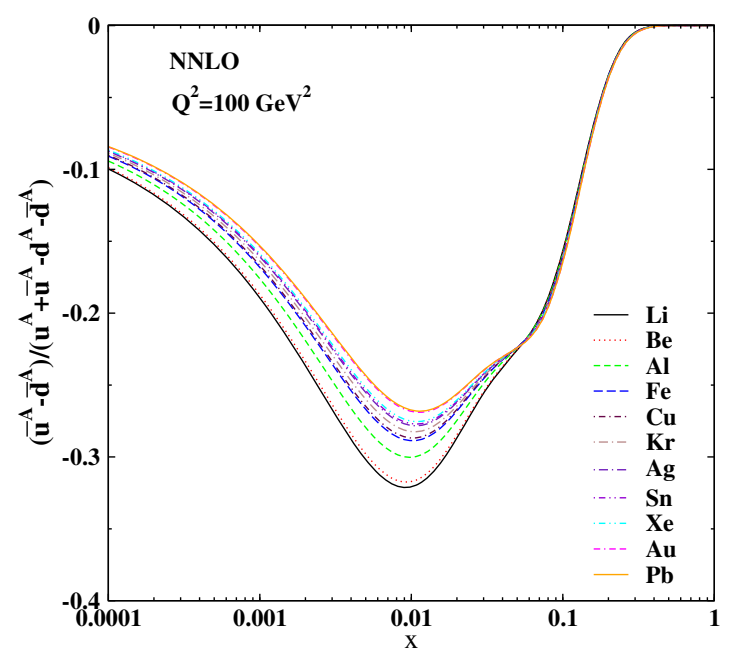

FIG. 9. The ratio of flavor asymmetric distributions, $\left(\bar{u}^{A}-\right.$ $\left.\bar{d}^{A}\right) /\left(u^{A}+\bar{u}^{A}-d^{A}-\bar{d}^{A}\right)$ is shown for some nuclei that have experimental data at $\mathrm{Q}^{2}=100 \mathrm{GeV}^{2}$. In the isoscalar nuclei, the distributions vanish, $\left(\bar{d}^{A}-\bar{u}^{A}=0\right)$.
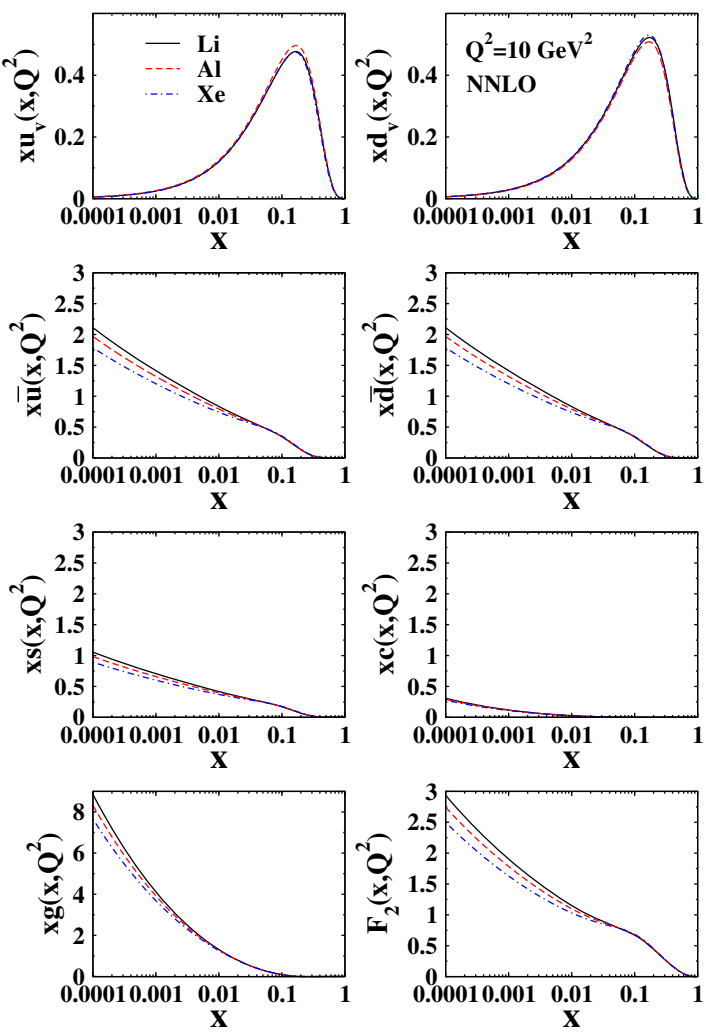

FIG. 10. Comparison between nuclear parton distributions in NNLO for a range of nuclei such as $\mathrm{Li}, \mathrm{Al}$, and Xe nucleus at the $\mathrm{Q}^{2}=10 \mathrm{GeV}^{2}$.

because we directly determine the $\bar{d}+\bar{u}$ combination from the analysis.

\section{COMPARISON WITH THE EXPERIMENTAL DATA}

A detailed comparison of our NNLO nuclear PDFs results with the experimental data used in this analysis is presented in this section. The error bars in the figures correspond to the statistical and systematic errors added in quadrature. As we mentioned, the available data are taken in the limited $x$ range without small $x$ data, which leads to difficulty in determining the nuclear gluon distribution. Figure 11 shows this issue. In this figure, we plot the theoretical prediction including uncertainties for structure function ratio of calcium nucleus, $F_{2}^{A(=C a)} / F_{2}^{D}$, which has been compared with actual data at $\mathrm{Q}^{2}=5 \mathrm{GeV}^{2}$. Our previous next-to-leading order nuclear PDFs analysis AT12 [22] and the results from HKNO7 [30] are also shown as well. The theoretical predictions are shown by the curves in the figure and the uncertainties are shown by the shaded bands. The plot shows that our NNLO parametrizations are successful in explaining the $x$ dependence of the calcium data as an example.

Figure 12 shows the ratio $R=F_{2}^{A}\left(x, Q^{2}\right) / F_{2}^{A^{\prime}}\left(x, Q^{2}\right)$ in comparison to NMC data for a variety of nuclear targets. 


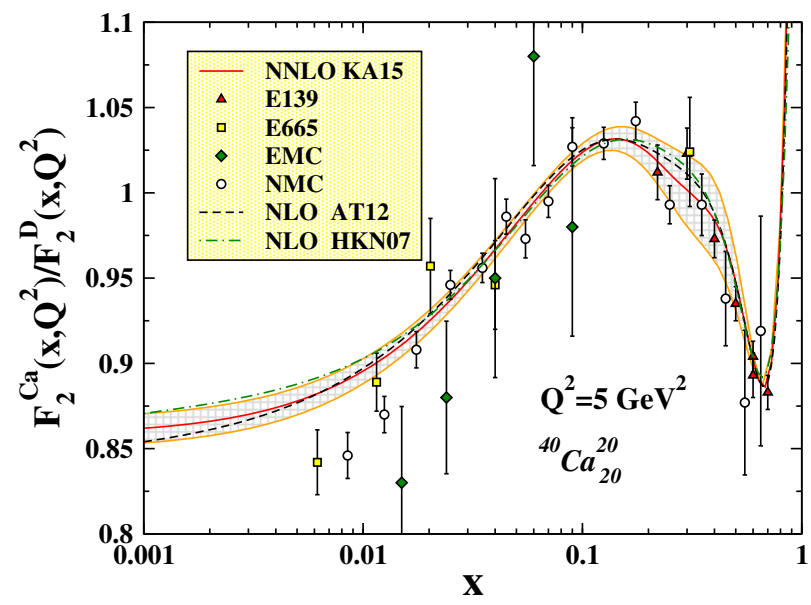

FIG. 11. EMC effect for calcium nucleus at $\mathrm{Q}^{2}=5 \mathrm{GeV}^{2}$ in NNLO approximation and its comparison with our previous NLO analysis [22]. The results from HKN07 [30] are also have been shown. In this plot, theoretical results are compared with the $F_{2}^{A(=C a)} / F_{2}^{D}$ data. The uncertainties are shown by the shaded bands.

The plot clearly shows that both KA15 NNLO and AT12 NLO theory predictions describe the data well.

A detailed comparison with the experimental data of the structure function ratios $R=F_{2}^{A} / F_{2}^{D}$ for the analyzed nuclei are shown in Figs. 13 and 14.

The ratios of $\left(R^{\text {data }}-R^{\text {theory }}\right) / R^{\text {theory }}$ are also shown for comparison. $R^{\text {data }}$ is the experimental value and $R^{\text {theory }}$ is the theoretical value of the structure function ratios. The same plots for the structure function ratios of $R=F_{2}^{A} / F_{2}^{C}$

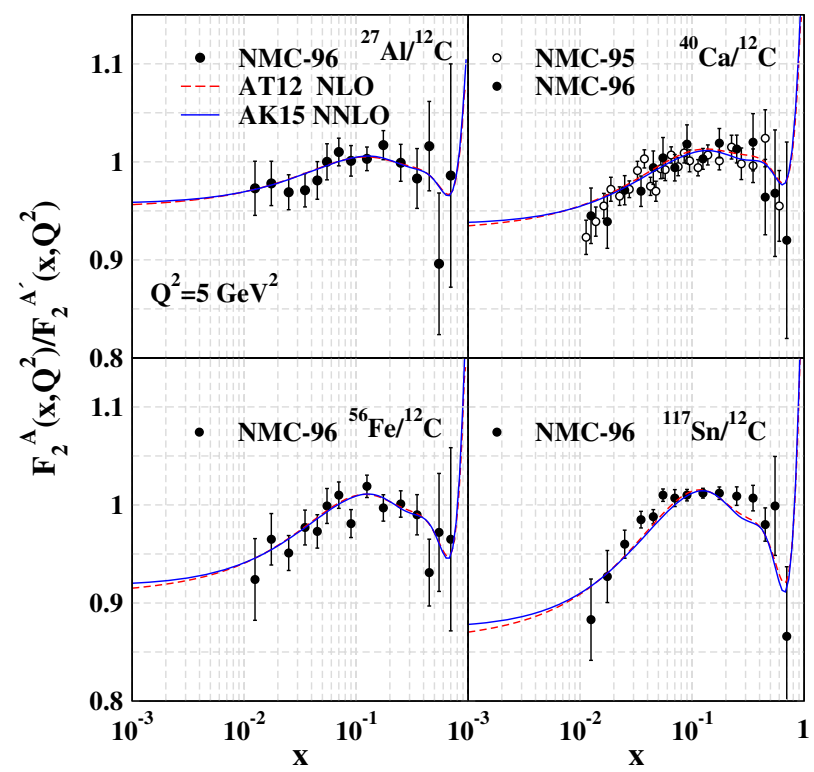

FIG. 12. Comparison of the KA15 NNLO and AT12 NLO theory predictions for $R=F_{2}^{A}\left(x, Q^{2}\right) / F_{2}^{A^{\prime}}\left(x, Q^{2}\right)$ as a function of $x$ at the scale $\mathrm{Q}^{2}=5 \mathrm{GeV}^{2}$. The data from NMC has been shown for comparison.
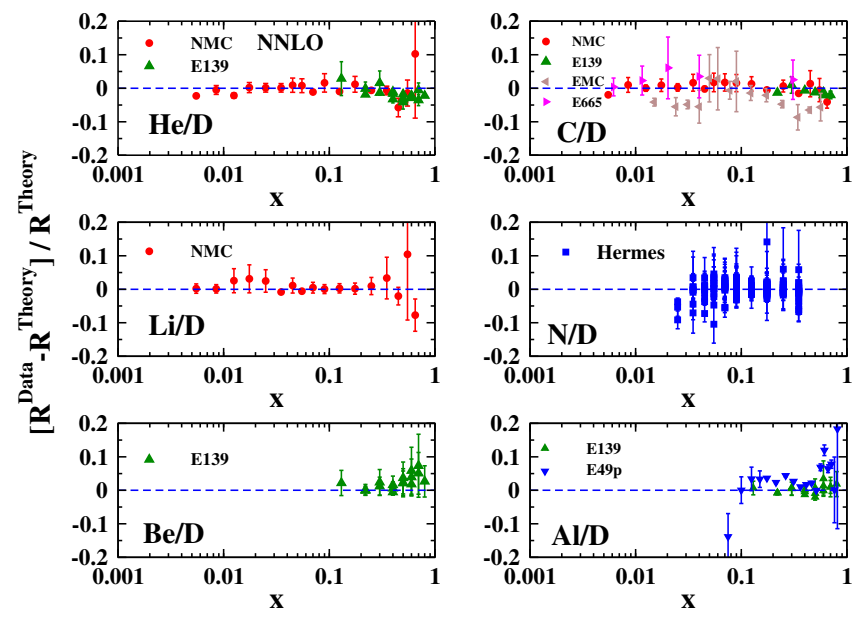

FIG. 13. Comparison with the experimental data of $R=F_{2}^{A} / F_{2}^{D}$. The ratios of $\left(R^{\text {data }}-R^{\text {theory }}\right) / R^{\text {theory }}$ are shown for comparison. The NNLO parametrization is used for the theoretical calculations at the $Q^{2}$ points of the experimental data.

and $R=F_{2}^{A} / F_{2}^{L i}$ are also have been shown in Fig. 15. The comparison indicates that our NNLO parametrizations should be successful in explaining the $x$ dependance of the analyzed nuclei experimental data.

In order to better investigate the nuclear PDFs, we plot the $\mathrm{Q}^{2}$ dependence of the structure function ratios $F_{2}^{S n} / F_{2}^{C}$ at NNLO in comparison with the experimental data of NMC-96 in Fig. 16. The comparisons are shown for some

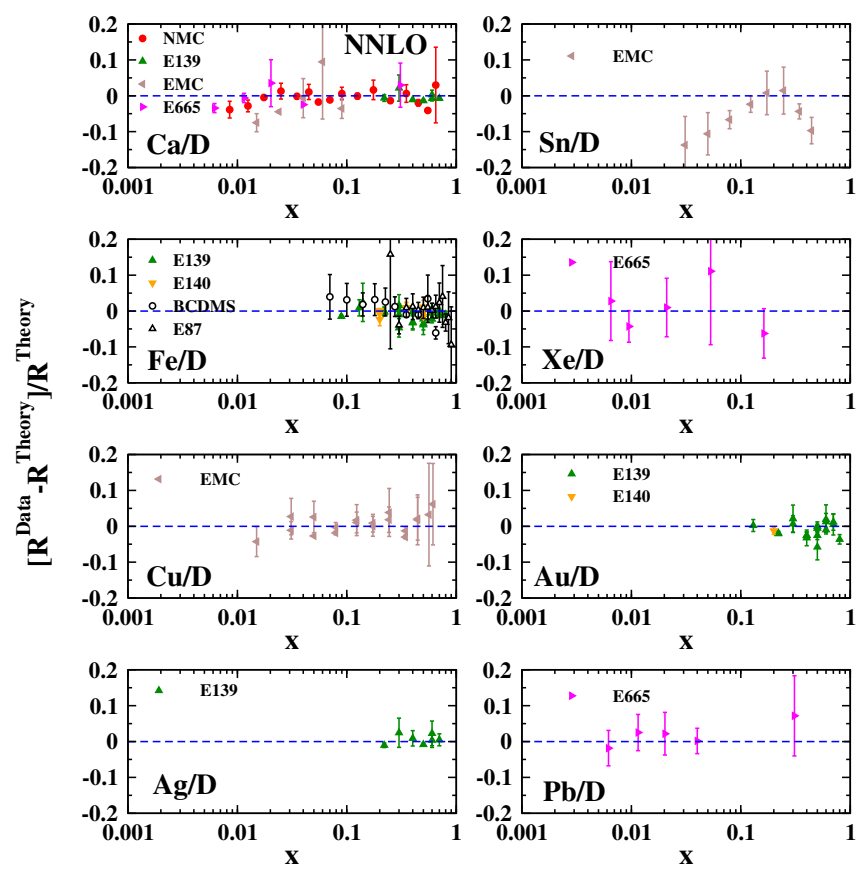

FIG. 14. Comparison with experimental data of $R=F_{2}^{A} / F_{2}^{D}$. The ratios of $\left(R^{\text {data }}-R^{\text {theory }}\right) / R^{\text {theory }}$ are shown for comparison. The NNLO parametrization is used for the theoretical calculations at the $Q^{2}$ points of the experimental data. 


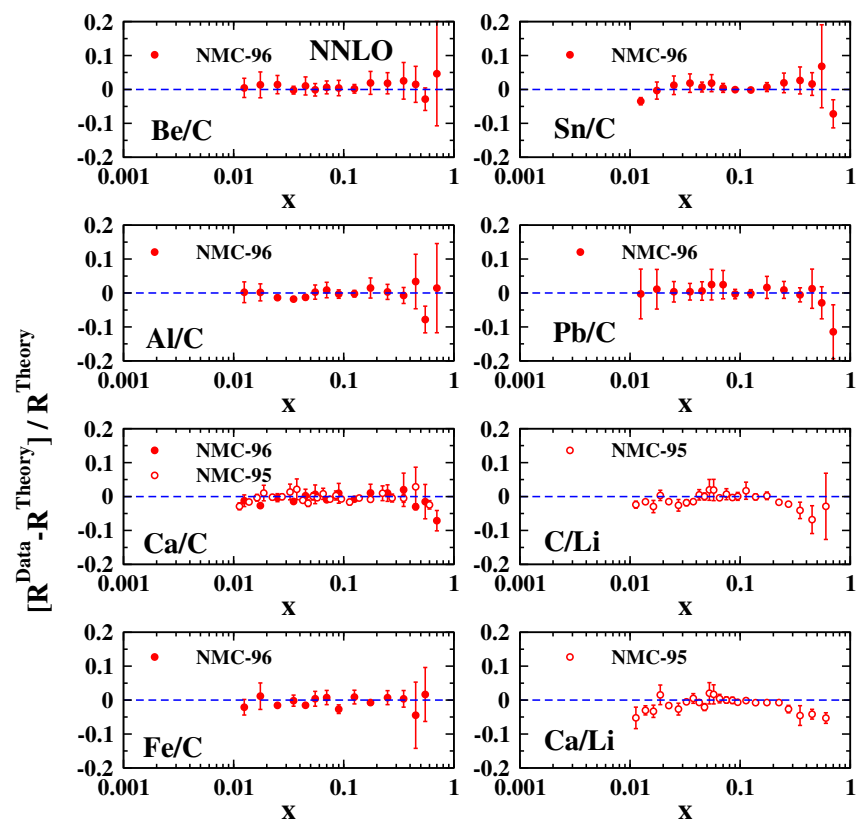

FIG. 15. Comparison with experimental data of $R=F_{2}^{A} / F_{2}^{C}$ and $R=F_{2}^{A} / F_{2}^{L i}$. The ratios of $\left(R^{\text {data }}-R^{\text {theory }}\right) / R^{\text {theory }}$ are shown for comparison. The NNLO parametrization is used for the theoretical calculations at the $Q^{2}$ points of the experimental data.

selected smaller values of $x, x=0.07,0.09,0.035,0.045$, and 0.055 . The results indicating the overall $\mathrm{Q}^{2}$ dependencies are in very good agreement with the data.

$\mathrm{Q}^{2}$ dependence of the theoretical predictions of the structure function ratios $F_{2}^{P b} / F_{2}^{D}$ at NNLO for different value of $x, x=0.001,0.01,0.01$, and 0.3 including their uncertainties have been shown in Fig. 17. The theoretical

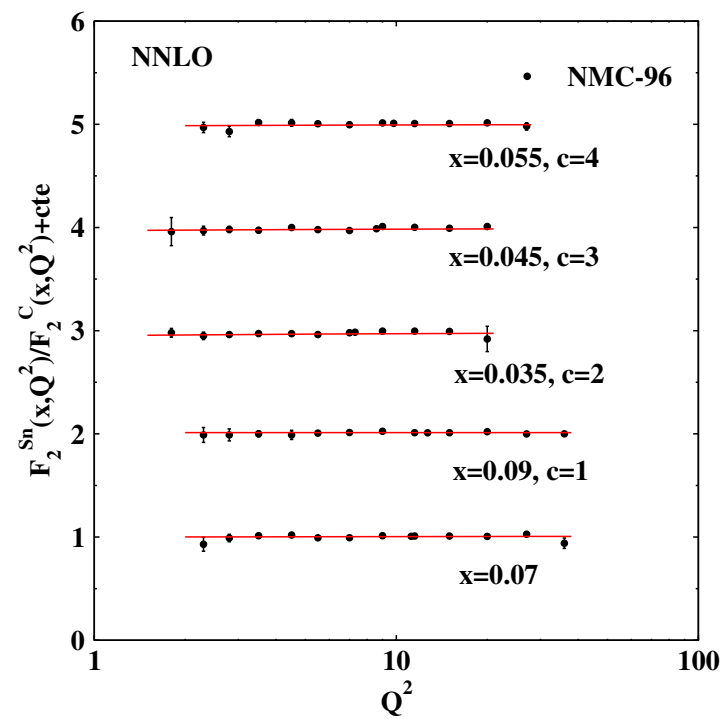

FIG. 16. The $\mathrm{Q}^{2}$ dependence of the structure function ratios $F_{2}^{S n} / F_{2}^{C}$ at NNLO in comparison with the experimental data of NMC-96.
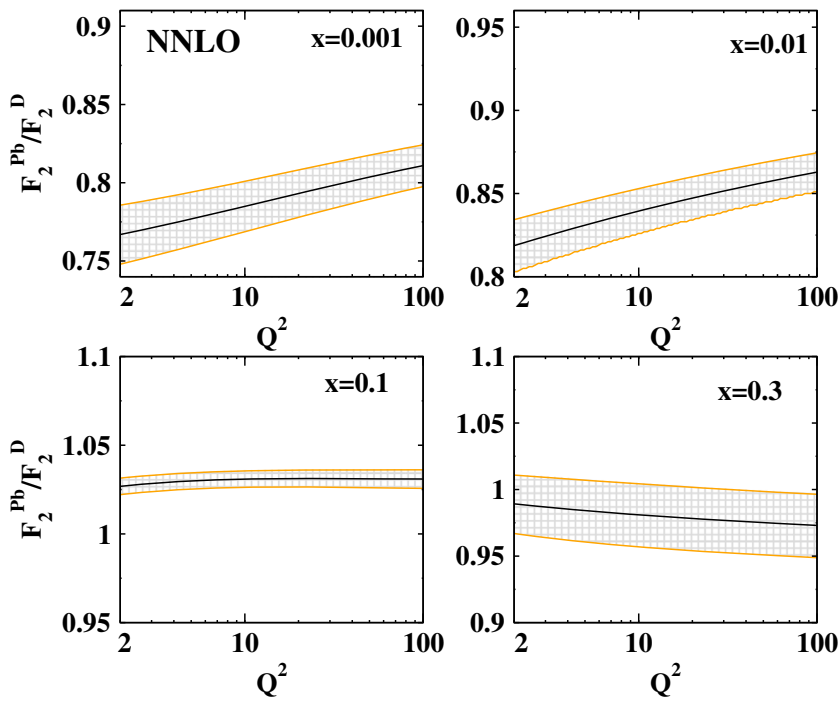

FIG. 17. $Q^{2}$ dependence of the theoretical predictions of the structure function ratios $F_{2}^{P b} / F_{2}^{D}$ at NNLO for different value of $x=0.001,0.01,0.01$, and 0.3 including their uncertainties.

predictions are shown by the curves in the figure and the uncertainties are shown by the shaded bands.

Using the Drell-Yan data of proton-nucleus scattering, one can investigate the nuclear modification of antiquark distributions. In Fig. 18, the theoretical predictions are compared with the data of the Drell-Yan cross-section ratios $\sigma_{\mathrm{DY}}^{\mathrm{Fe}} / \sigma_{\mathrm{DY}}^{\mathrm{Be}}$ measured by FNAL-E866 [74] at
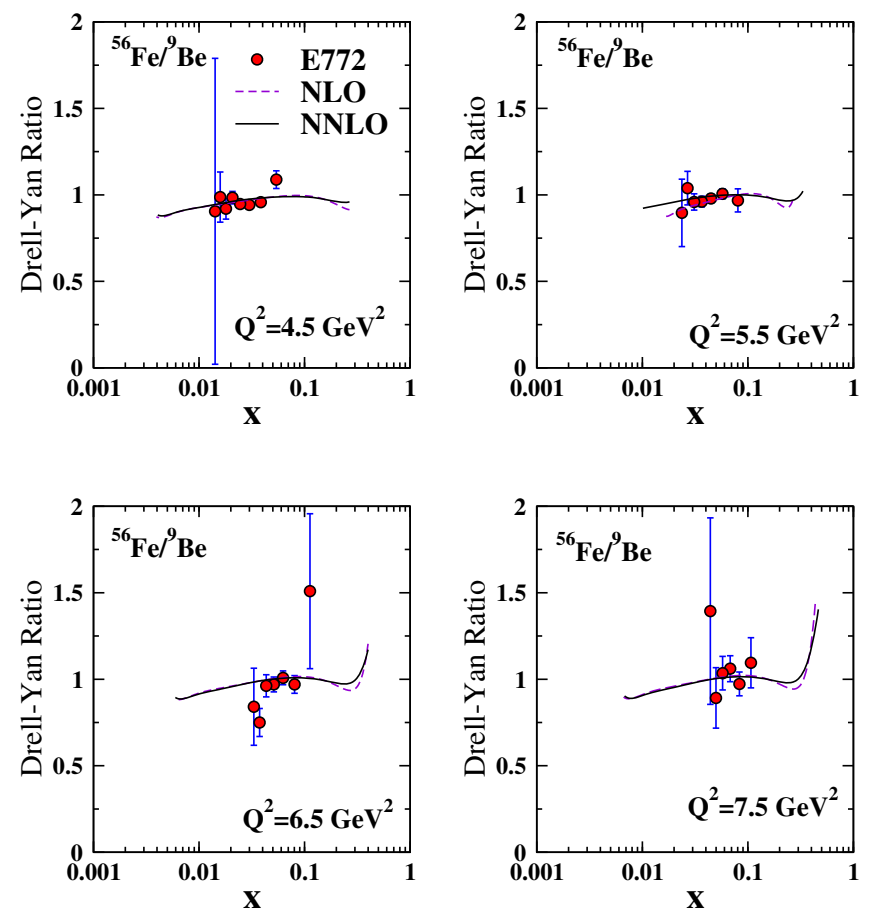

FIG. 18. Theoretical predictions are compared with the data of the Drell-Yan cross-section ratios $\sigma_{\mathrm{DY}}^{\mathrm{Fe}} / \sigma_{\mathrm{DY}}^{\mathrm{Be}}$. Data points are from the FNAL-E866 [74] experiments at Fermilab. 
$\mathrm{Q}^{2}=4.5 \mathrm{GeV}^{2}, 5.5 \mathrm{GeV}^{2}, 6.5 \mathrm{GeV}^{2}$, and $7.5 \mathrm{GeV}^{2}$. Our previous results for nuclear PDFs at NLO are also shown as well.

The FNAL-E866 data on Drell-Yan cross-section are in a good agreement with the AT12 NLO and KA15 NNLO predictions. The cross section of the Drell-Yan process is too small to study any process with colliding ion beams at higher center-of-mass energies. As we mentioned, the data from proton-nucleus and proton-deuteron collisions at the CERN-LHC or RHIC would be very desirable in order to determine the nuclear PDFs at low values of parton fractional momenta $x[91,92]$.

\section{COMPARISON WITH DIFFERENT GLOBAL ANALYSES OF NUCLEAR PDFS}

We are now in position to compare our NNLO nuclear PDFs KA15 with other recent nuclear parton distributions in the literature. Specifically, we will compare our results with the following nuclear PDFs sets: AT12 [22], EPSO 9 [27], HKNO 7 [30], nDS [36], and DSSZ12 [39]. We will briefly summarize the key development of the most recent ones of these. The initial scale in EPSO9 [27] is set to $\mathrm{Q}_{0}=1.3 \mathrm{GeV}$ and it uses the CTEQ6.1M free proton NLO PDFs. The ZM-VFNS heavy-quark scheme are adopted and the data from $\ell+A$ DIS and $\mathrm{p}+\mathrm{A}$ DY and $\pi^{0}$ production in $\mathrm{d}+\mathrm{Au}$ collisions at PHENIX are used in the EPSO9 nuclear PDFs analysis. The DSSZ12 [39] uses the free proton NLO PDFs of MSTW, consequently the nuclear modification factors are parametrize at $\mathrm{Q}_{0}=1 \mathrm{GeV}$. Heavy quarks effects are included using general-mass variable-flavor number scheme (GMVFNS). This analysis covers the most extensive selection of the nuclear data including $\ell^{ \pm}$-DIS data, $\mathrm{p}+\mathrm{A}$ DY data together with $\nu$-DIS and $\pi^{0}$ production in $\mathrm{d}+\mathrm{Au}$ collisions from PHENIX and STAR. The latest HKN07 global analysis of nuclear PDFs presented in [30] uses the MRST98 NLO parametrization for the nucleonic PDFs. This analysis covers the $\ell+A$ DIS and $\mathrm{p}+\mathrm{A}$ DY data. Charm quark contributions are included and the strange quark contributions are assumed to be symmetric.

A detailed comparison of different approach resulting from the available nuclear PDFs analyses can be found in Fig. 19. The plots show that the differences are noticeable. For almost all PDFs at $\mathrm{Q}^{2}=10 \mathrm{GeV}^{2}$ shown for Fe in the figure, our NLO and NNLO nuclear PDFs have significant overlap with HKNO 7 through much of the $x$ range. It is due to that that the technical framework and data set selection of our global analysis are closest to HKN07 nuclear PDFs analysis. For the $u$ and $d$ PDFs, both our NLO and NNLO results including $\mathrm{HKNO} 7$ show a stronger shadowing suppression at small values of $x$. In medium to small $x$ of $\bar{u}, \bar{d}$ and $s$ PDFs, we have slight overlap with other nuclear PDFs sets. For the gluon PDF, there is a variation among the different PDFs sets. The AT12, HKN07,
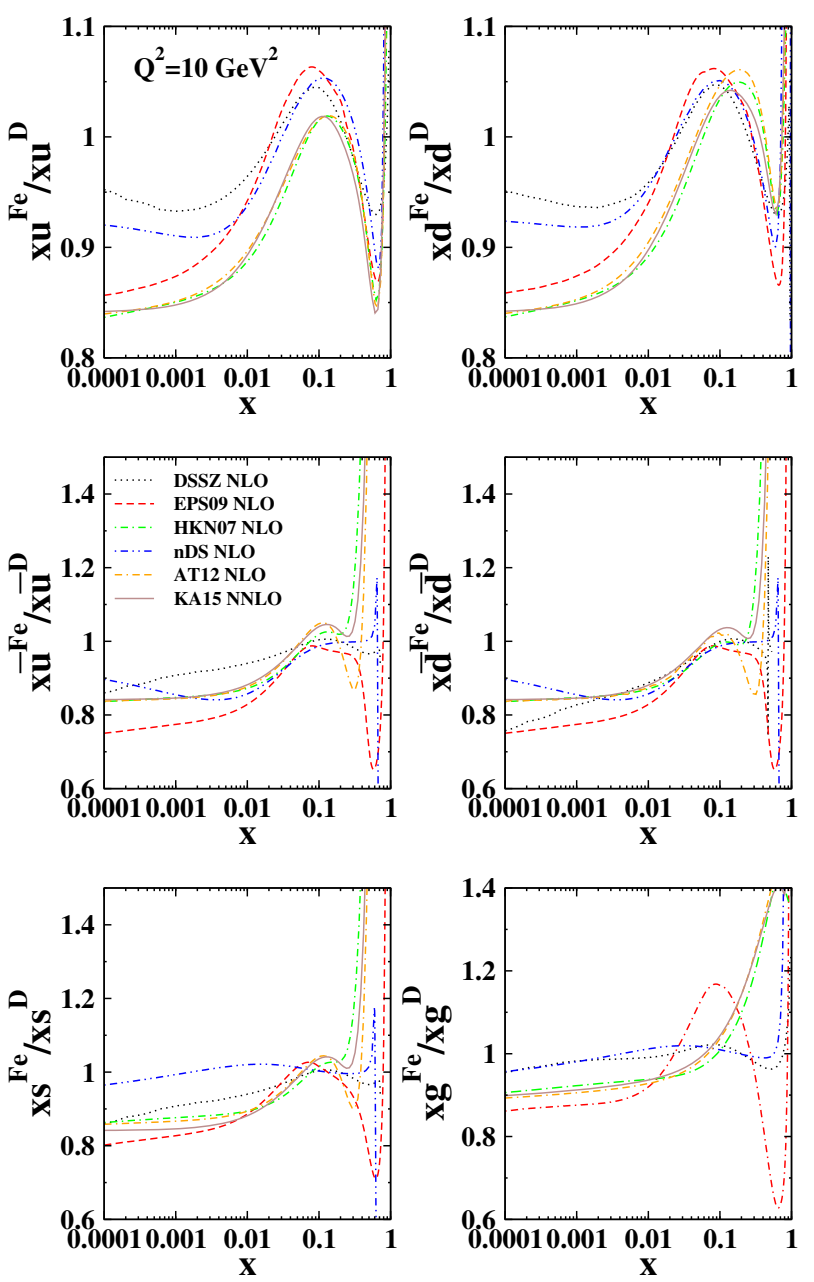

FIG. 19. The obtained NNLO nuclear modification factors, KA15, defined in Eq. (3) as a function of $x$ for iron at $\mathrm{Q}^{2}=10 \mathrm{GeV}^{2}$. The results from other groups such as $\mathrm{AT} 12$ [22], EPSO 9 [27], HKN07 [30], nDS [36], and DSSZ [39] have been shown for comparison.

EPS09, and KA15 $g$ PDFs all agree very nicely with each other throughout the medium to small $x,(0.001 \lesssim$ $x \lesssim 0.1)$. However the EPSO 9 shows stronger shadowing suppression at small values of $x$. It has also a larger enhancement in the antishadowing region $(\mathrm{x} \sim 0.1)$. The DSSZ12 and nDS gluon PDFs agree nicely throughout much of the $x$ range.

In Figs. 20 and 21, we plot nuclear modifications for the nuclear PDFs of a proton bound in gold and lead respectively. We show the results for these rather heavy nuclei, because they are the main targets at the heavy ion colliders such as LHC. The ratios are plotted as a function of $x$ at the scale $\mathrm{Q}^{2}=5 \mathrm{GeV}^{2}$. The results from HKN07 and EPSO 9 global nuclear PDFs analyses are also have been shown for comparison. For the $u, d$, and $s$ PDFs, we have overlap with HKN0 7 results while the nuclear gluon PDFs has larger shadowing suppression at small values of $x$ than HKN0 7 analysis. 

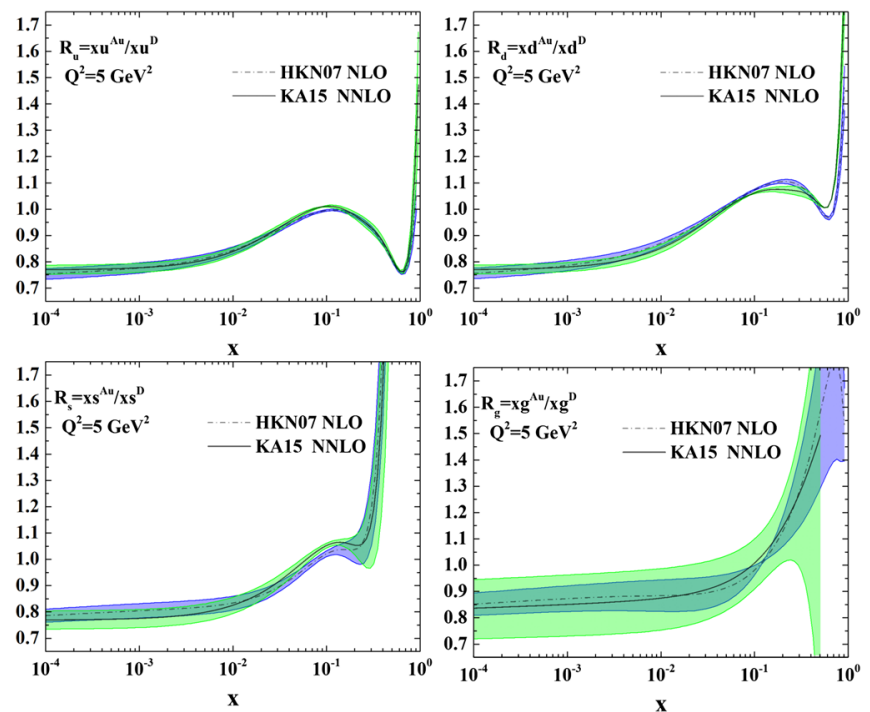

FIG. 20. Comparison of the KA15 fit (green) with the results obtained by HKN07 (blue) [30]. The ratios are plotted as a function of $x$ at the scale $\mathrm{Q}^{2}=5 \mathrm{GeV}^{2}$ for a gold nucleus. The error bands show the uncertainty of the nuclear PDFs.

In comparison with EPSO9 which has been shown in Fig. 21, the uncertainty bands for all nuclear PDFs of our NNLO analysis are considerably smaller than the uncertainty band of EPSO 9 throughout much of the $x$ range.

In Fig. 22, we plot the $s$ and gluon PDFs as a function of $x$ at the scale $\mathrm{Q}^{2}=10 \mathrm{GeV}^{2}$ for the lead nuclei. As we mentioned in Eq. (7), we relate the strange distribution to the light quarks sea $(\bar{u}$ and $\bar{d})$ distributions, consequently the $s$ distribution does not contribute in the fitting processes directly. As a result the strange distribution $s$ is similar to
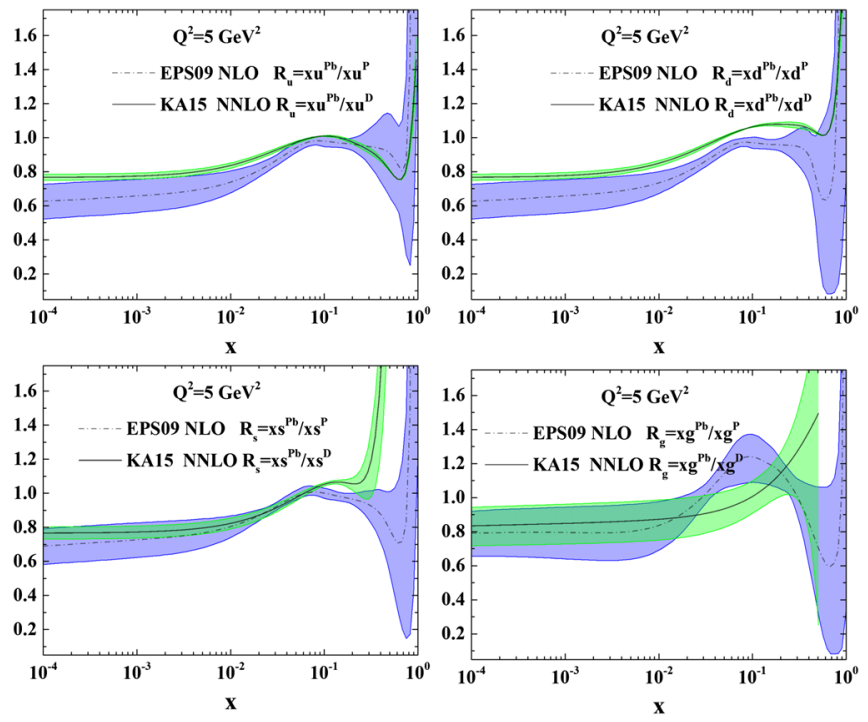

FIG. 21. Comparison of the KA15 fit (green) with the results obtained by EPS09 (blue) [27]. The ratios are plotted as a function of $x$ at the scale $\mathrm{Q}^{2}=5 \mathrm{GeV}^{2}$ for a lead nucleus. The error bands show the uncertainty of the nuclear PDFs.
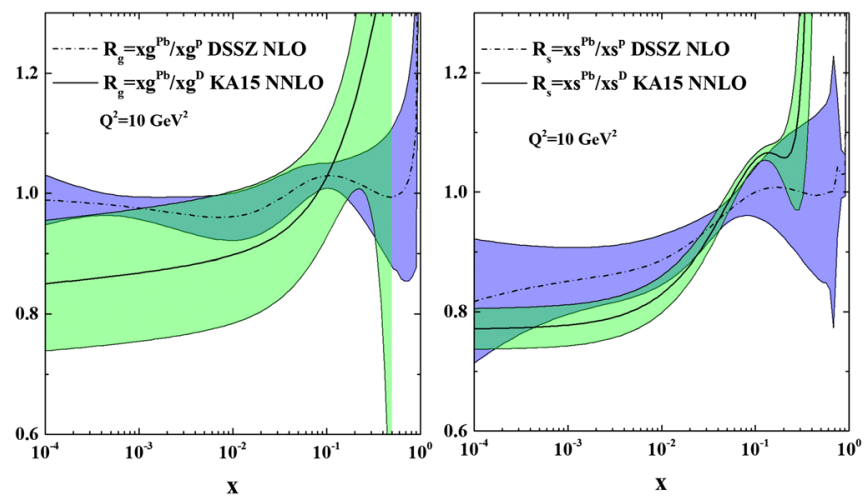

FIG. 22. Comparison of the KA15 fit (green) with the results obtained by DSSZ (blue) [24]. The ratios are plotted as a function of $x$ at the scale $\mathrm{Q}^{2}=10 \mathrm{GeV}^{2}$ for the lead nuclei. The error bands show the uncertainty of the nuclear PDFs.

the light quarks sea distributions. This behavior leads to considerably smaller uncertainties for the strange distribution. The DSSZ12 assume that the light strange quark $s$ and antiquark $\bar{s}$ have the same modification factors and relate them to the valance quarks modification factors. As Fig. 22 shows, the DSSZ12 light strange quark distribution has bigger uncertainties both for small and large $x$. For the gluon distribution, this treatment is rather different. The DSSZ12 analysis shows a better description of EMC effect and also smaller uncertainty band. The gluon shadowing in the small $x$ region $(x \lesssim 0.01)$ has been constrained by the momentum sum rule and indirectly by the $\mathrm{Q}^{2}$ evolution effects in the sea quarks sector which reflected by the DIS and Drell-Yan (DY) data. In addition, the inclusion of new and more precise measurements for example high- $p_{T}$ data from RHIC will provide important further constraints for the gluon shadowing region. The obtained gluon PDFs from KA15 analysis shows a stronger gluon shadowing at small $x$.

The mentioned differences between available nuclear PDFs analyses presented in this section generally arise from two sources, the selection of data points used in the global analysis and direct parametrization of the nuclear PDFs or parametrization of nuclear modifications factors. Overall we found relatively good agreements between different nuclear PDFs sets.

\section{NUCLEAR PDFS AT THE LHC ERA}

The proton + lead $(\mathrm{p}-\mathrm{Pb})$ and lead + lead $(\mathrm{Pb}-\mathrm{Pb})$ collisions are an integral part of the present and future nuclear programs at the Large Hadron Collider (LHC). As we mentioned, the nuclear PDFs are essential tools in high energy heavy-ion nucleus-nucleus (A-A) collisions at the future RHIC and CERN-LHC programs. Generally speaking, the nuclear PDFs has very important role in the ongoing LHC proton + lead and lead + lead collisions. Some works have been done in this regard to conclusively 


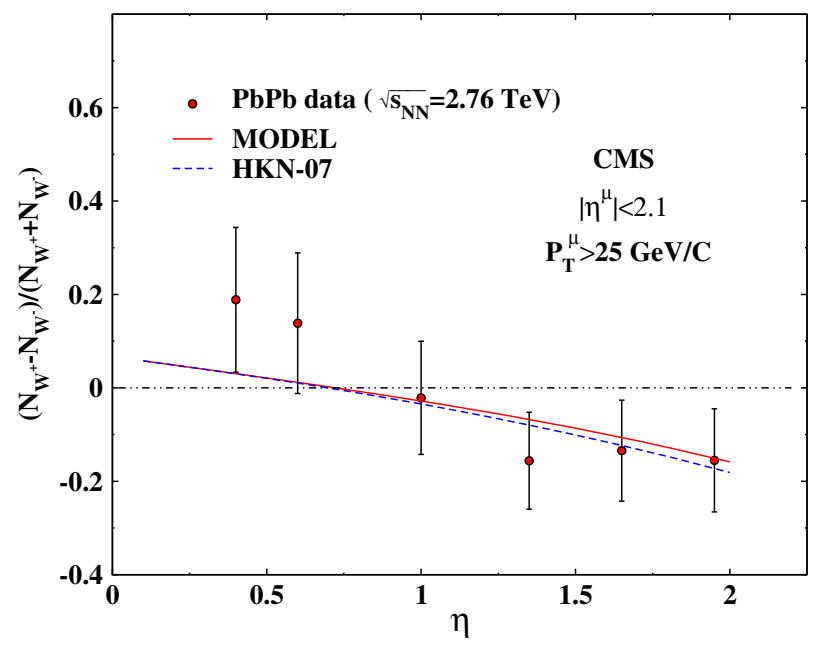

FIG. 23. Lepton $(\ell=\mu)$ charge asymmetry as a function of muon pseudorapidity for $\mathrm{Pb}-\mathrm{Pb}$ collisions recorded by CMS at $\sqrt{s_{N N}}=2.76 \mathrm{TeV}$ [111]. The red circles represent the data and the solid curve represent our theoretical predictions.

test the universality of the nuclear PDFs and also to investigate the sensitivity of the nuclear modifications in the PDFs [50,93-95]. The first experimental results published by the ALICE and CMS collaborations for the proton-lead $(\mathrm{p}-\mathrm{Pb})$ collisions at a nucleon-nucleon centerof-mass energy of $\sqrt{s_{N N}}=5.02 \mathrm{TeV}$ are summarized in details in Refs. $[96,97]$. The CMS Collaboration also has recorded $150 \mu b^{-1}$ in $\mathrm{Pb}-\mathrm{Pb}$ collisions at $\sqrt{s_{N N}}=$ $2.76 \mathrm{TeV}$ [98]. All the heavy-ion public physics results from the CMS Collaboration are collected in Ref. [99]. Some experimental studies on different aspects of heavyion collisions are presented in Refs. [100-113].

$\mathrm{W}$ and $\mathrm{Z}$ boson production in proton-nucleus $(\mathrm{p}-\mathrm{A})$ and nucleus-nucleus (A-A) collisions at the CERN-LHC offer a unique opportunity to probe nuclear PDFs. The CMS collaboration at CERN presented their first study on $\mathrm{W}$ production (via leptonic decay channel) in $\mathrm{Pb}-\mathrm{Pb}$ collisions at $\sqrt{s_{N N}}=2.76 \mathrm{TeV}$ [111] and in $\mathrm{p}-\mathrm{Pb}$ collisions at $\sqrt{s_{N N}}=5.02 \mathrm{TeV}$ [110] and also the $\mathrm{Z}$ boson production (via dimuon and dielectron decay channels) in $\mathrm{Pb}-\mathrm{Pb}$ collisions at $\sqrt{s_{N N}}=2.76 \mathrm{TeV}[114,115]$. From discussion that we made in the paper, more data are needed to constrain the nuclear PDFs. The main difficulty of all global analysis of nuclear PDFs is the lack of any DIS data with heavy-ion beams which lead to larger uncertainties. For this reason, the obtained nuclear PDFs are less precisely known for nuclei than for the nucleons. As a consequence, precise measurements of the $\mathrm{W}$ boson production in heavy-ion collisions and including the corresponding data in any global fits may lead to an improved determination of the nuclear PDFs [110]. Moreover, lepton charge asymmetry via dominant processes at the LHC in $\mathrm{W}^{+}\left(u \bar{d} \rightarrow W^{+} \rightarrow \ell^{+} \nu_{\ell}\right)$ and $\mathrm{W}^{-}\left(\bar{u} d \rightarrow W^{-} \rightarrow \ell^{-} \nu_{\ell}\right)$ productions, can permit the flavor asymmetries of $d$ and $u$ quark distributions in the nuclei. The lepton (muon) charge asymmetry in $\mathrm{Pb}-\mathrm{Pb}$ collisions collected by the CMS experiment at $\sqrt{s_{N N}}=2.76 \mathrm{TeV}$ [111] is shown in Fig. 23 and compared to our theoretical predictions. The theoretical results from HKNO7 [30] are also shown as well.

In addition to the lepton charge asymmetry, study on the nuclear modification factor of the PDFs is the current interest in the recent heavy-ion collisions at CERN-LHC $[112,114,116,117]$. The present measurements may set significant constraints for the global fits of the nuclear PDFs in an unexplored kinematical region of $x$.

Furthermore, recent detailed studies show the possibilities of direct measurements of large-mass elementary particles such as Higgs boson and top-quark via heavyion collision at the multi-TeV CERN-LHC and proposed future circular collider (FCC) [118,119]. Double-top or single-top productions in lead-lead $(\mathrm{Pb}-\mathrm{Pb})$ and protonlead $(\mathrm{p}-\mathrm{Pb})$ collisions can be used to constrain the nuclear PDFs, especially the nuclear gluon distribution in small value of momentum fraction, $x \approx 10^{-3}-10^{-2}$. Our study on the single and pair-production of top-quark at LHC and FCC energies via $\mathrm{p}-\mathrm{Pb}$ and $\mathrm{Pb}-\mathrm{Pb}$ collisions is in progress.

\section{SUMMARY AND CONCLUSIONS}

We presented, for the first time, a global analysis of nuclear PDFs and their uncertainties at the next-to-nextto-leading order (NNLO) accuracy in perturbative QCD. We performed a $\chi^{2}$ analysis using available DIS $\ell^{ \pm}+$ nucleus and Drell-Yan data. The uncertainties of the determined nuclear PDFs are estimated using the Hessian method. The nuclear charm quarks distributions are also added into the analysis. The result of the fit is a set of nuclear PDFs which incorporate the $x, Q$ and also A dependence, so one can accommodate the full range of nuclear targets from light $\mathrm{A}=2$ to heavy $\mathrm{A}=208$. A good fit to data has been obtained. We find a good agreement with experimental data and other fits. As new and more precise measurements of observables sensitive to the gluon distribution will become available in the future high energy experiments, we are expecting smaller uncertainty on the fitted nuclear PDFs. In this respect, data from the CERN-LHC proton-lead run including dijet data from the CMS collaboration, are foreseen to bring significant additional insight. It will also provide us a new window in theoretical understanding of the high energy process involving nucleons. There are large amount of precise data for the free proton case, so one can develop a combined analysis of proton PDFs and nuclear PDFs. The combination of PDFs and nuclear PDFs analysis provide good constraints on the gluon distributions at small values of Bjorken- $x$ and may allow for a good separation of the quark flavours in a wide range of $x$, which are mostly important for the present and future 
collider phenomenological tasks. The new measurements of the nuclear effects in the Drell-Yan production which is planned in the E906/Drell-Yan experiment at Fermilab [120-122] would be interested to analysis. The primary goals of this measurement at Fermilab include the study of the anti- $d$ to anti- $u$ quark asymmetry in the proton and a detailed study of the EMC effect in sea quarks. Our next goal is to perform the present analysis, as well as, when the mentioned data become finalized, including upcoming heavy-ion collisions data sets from CERN-LHC [119] and photon production in $\mathrm{d}+\mathrm{Au}$ and $\mathrm{Au}+\mathrm{Au}$ from PHENIX [123-125]. Selecting a complete data set plays a major role in constraining the nuclear modifications in any nuclear PDFs analysis. Further constraints for the nuclear gluon distributions in the yet unexplored regions of the $x$ and $\mathrm{Q}^{2}$ plane are absolutely necessary for understanding QCD parton dynamics in hadronic and nuclear high-energy collisions. Our next-to-next-to-leading order of nuclear PDFs including their uncertainties can be calculated using the codes discussed in Appendix B.

\section{ACKNOWLEDGMENTS}

The authors are especially grateful to Fredrick Olness from SMU for fruitful discussions and critical remarks. We also would like to thank Mojtaba Mohammadi Najafabadi, Abolfazl Mirjalili, and Muhammad Goharipour for carefully reading the manuscript and their helpful comments. We are also grateful to Rodolfo Sassot and Pia Zurita for providing us with the best fit of DSSZ12 nuclear PDFs. Authors are thankful School of Particles and Accelerators, Institute for Research in Fundamental Sciences (IPM) for financially support of this project. H. K. also thanks the University of Science and Technology of Mazandaran for financial support provided for this research.

\section{APPENDIX A: SUM RULES, BARYON NUMBER AND MOMENTUM CONSERVATION}

Using three sum rules presented in Eq. (6) which give us the nuclear charge $Z$, baryon number $A$, and momentum conservation, one can calculate the three parameters $a_{u_{v}}(A, Z), a_{d_{v}}(A, Z)$, and $a_{g}(A, Z)$. For practical usage, we express these constants by eight integral values $I_{1}-I_{8}$ as we explained in our previous version of nuclear PDFs [22]:

TABLE III. Numerical values of the eight integrals for lead.

\begin{tabular}{lccc}
\hline \hline Integral & Value & Integral & Value \\
\hline $\mathrm{I}_{1}$ & 0.0890676 & $\mathrm{I}_{5}$ & 0.374181 \\
$\mathrm{I}_{2}$ & 0.0537472 & $\mathrm{I}_{6}$ & 0.156111 \\
$\mathrm{I}_{3}$ & 2.1693 & $\mathrm{I}_{7}$ & 0.0226629 \\
$\mathrm{I}_{4}$ & 1.06856 & $\mathrm{I}_{8}$ & 0.39874 \\
\hline \hline
\end{tabular}

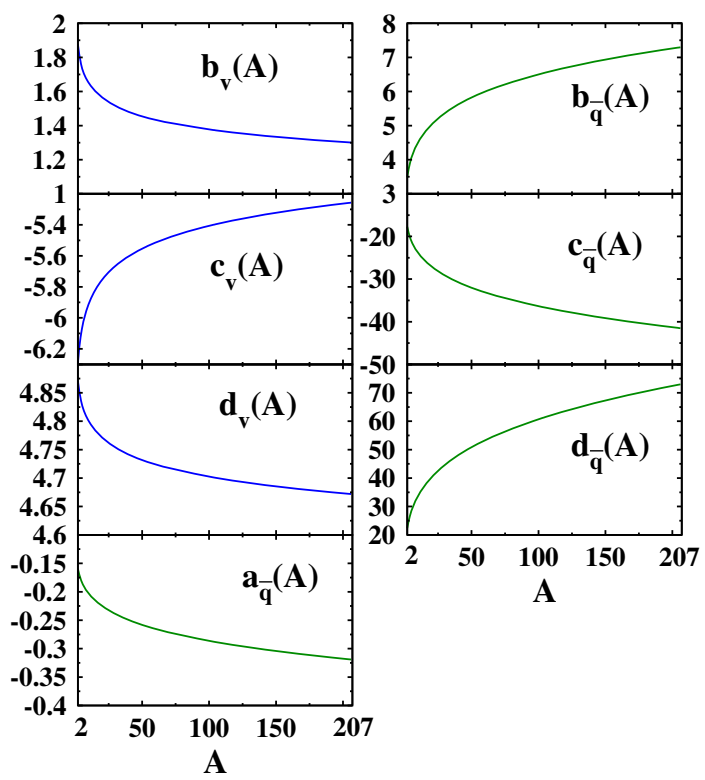

FIG. 24. A-dependence of the fit parameters according to Eq. (3).

$$
\begin{aligned}
a_{u_{v}}(A, Z)= & -\frac{Z I_{1}(A)+(A-Z) I_{2}(A)}{Z I_{3}+(A-Z) I_{4}}, \\
a_{d_{v}}(A, Z)= & -\frac{Z I_{2}(A)+(A-Z) I_{1}(A)}{Z I_{4}+(A-Z) I_{3}}, \\
a_{g}(A, Z)= & -\frac{1}{I_{8}}\left\{a_{u_{v}}(A, Z)\left[\frac{Z}{A} I_{5}+\left(1-\frac{Z}{A}\right) I_{6}\right]\right. \\
& \left.+a_{d_{v}}(A, Z)\left[\frac{Z}{A} I_{6}+\left(1-\frac{Z}{A} I_{5}\right)\right]+I_{7}(A)\right\} .
\end{aligned}
$$

The numerical values of the eight integrals are listed in Table III from the present analysis for lead.

Using these values together with Eq. (A1), one could calculate the constants: $a_{u_{v}}=-0.0450391, a_{d_{v}}=-0.0433013$ and $a_{g}=0.00208932$, for lead. The A-dependence of the parameters in Eq. (3) is plotted in Fig. 24.

\section{APPENDIX B: FORTRAN PACKAGE OF KA15 NUCLEAR PDFS}

We prepared a code for calculating the nuclear PDFs including their uncertainties at different values of $x$ and $\mathrm{Q}^{2}$. The FORTRAN package containing our unpolarized structure functions, $F_{2}^{(A, Z)}\left(x, Q^{2}\right)$, for nuclei as well as the nuclear parton densities $x u_{v}^{A}\left(x, Q^{2}\right), \quad x d_{v}^{A}\left(x, Q^{2}\right), \quad x \bar{u}^{A}\left(x, Q^{2}\right)$, $x \bar{d}^{A}\left(x, Q^{2}\right), x s^{A}\left(x, Q^{2}\right), x c^{A}\left(x, Q^{2}\right), x g^{A}\left(x, Q^{2}\right)$ and their uncertainties at NNLO approximation in the $\overline{\mathrm{MS}}$-scheme can be obtained via e-mail from the authors. In this package we assumed the following kinematical ranges $10^{-4} \leq x \leq$ 0.999 and $1 \leq Q^{2} \leq 10^{5} \mathrm{GeV}^{2}$ for the $x$ and $\mathrm{Q}^{2}$ respectively. The obtained nuclear PDFs can be used for high-energy nuclear reactions to study the nuclear effects. 
[1] A. Buckley, J. Ferrando, S. Lloyd, K. Nordström, B. Page, M. Rüfenacht, M. Schönherr, and G. Watt, LHAPDF6: Parton density access in the LHC precision era, Eur. Phys. J. C 75, 132 (2015).

[2] L. A. Harland-Lang, A. D. Martin, P. Motylinski, and R. S. Thorne, Parton distributions in the LHC era: MMHT 2014 PDFs, Eur. Phys. J. C 75, 204 (2015).

[3] H. Khanpour, A. N. Khorramian, and S. Atashbar Tehrani, Parton distribution functions from QCD analysis of HERA combined inclusive $e^{ \pm} p$ scattering cross section data, Few Body Syst. 52, 279 (2012).

[4] H. Khanpour, A. N. Khorramian, and S. A. Tehrani, New parton distributions in fixed flavour factorization scheme from recent deep-inelastic-scattering data, J. Phys. G 40, 045002 (2013).

[5] S. Alekhin, J. Blumlein, and S. Moch, Parton distribution functions and benchmark cross sections at NNLO, Phys. Rev. D 86, 054009 (2012).

[6] F. D. Aaron et al. (H1 and ZEUS Collaborations), Combined measurement and QCD analysis of the inclusive $e^{ \pm} p$ scattering cross sections at HERA, J. High Energy Phys. 01 (2010) 109.

[7] P. Belov et al. (HERAFitter developers' Team Collaboration), Parton distribution functions at LO, NLO and NNLO with correlated uncertainties between orders, Eur. Phys. J. C 74, 3039 (2014)

[8] W. K. Tung, H. L. Lai, A. Belyaev, J. Pumplin, D. Stump, and C. P. Yuan, Heavy quark mass effects in deep inelastic scattering and global QCD analysis, J. High Energy Phys. 02 (2007) 053.

[9] H. L. Lai, M. Guzzi, J. Huston, Z. Li, P. M. Nadolsky, J. Pumplin, and C.P. Yuan, New parton distributions for collider physics, Phys. Rev. D 82, 074024 (2010).

[10] A. D. Martin, W. J. Stirling, R. S. Thorne, and G. Watt, Uncertainties on $\alpha_{S}$ in global PDF analyses and implications for predicted hadronic cross sections, Eur. Phys. J. C 64, 653 (2009).

[11] A. D. Martin, W. J. Stirling, R. S. Thorne, and G. Watt, Heavy-quark mass dependence in global PDF analyses and 3- and 4-flavour parton distributions, Eur. Phys. J. C 70, 51 (2010).

[12] A. D. Martin, W. J. Stirling, R. S. Thorne, and G. Watt, Parton distributions for the LHC, Eur. Phys. J. C 63, 189 (2009).

[13] S. Alekhin, J. Blumlein, S. Klein, and S. Moch, The 3-, 4-, and 5-flavour NNLO parton from deep-inelastic-scattering data and at hadron colliders, Phys. Rev. D 81, 014032 (2010).

[14] M. Gluck, P. Jimenez-Delgado, and E. Reya, Dynamical parton distributions of the nucleon and very small-x physics, Eur. Phys. J. C 53, 355 (2008).

[15] A. M. Cooper-Sarkar, HERAPDF fits including $F_{2}$ (charm) data, Proc. Sci., DIS2010 (2010) 023.

[16] S. Alekhin, J. Blumlein, and S. Moch, PDF fit in the fixedflavor-number scheme, Nucl. Phys. B, Proc. Suppl. 222-224, 41 (2012).

[17] W. K. Tung, H. L. Lai, A. Belyaev, J. Pumplin, D. Stump, and C. P. Yuan, Heavy quark mass effects in deep inelastic scattering and global QCD analysis, J. High Energy Phys. 02 (2007) 053.
[18] A. D. Martin, W. J. Stirling, R. S. Thorne, and G. Watt, Update of parton distributions at NNLO, Phys. Lett. B 652 , 292 (2007).

[19] P. Jimenez-Delgado and E. Reya, Dynamical NNLO parton distributions, Phys. Rev. D 79, 074023 (2009).

[20] J. F. Owens, A. Accardi, and W. Melnitchouk, Global parton distributions with nuclear and finite- $Q^{2}$ corrections, Phys. Rev. D 87, 094012 (2013).

[21] K. Kovarik et al., nCTEQ15-Global analysis of nuclear parton distributions with uncertainties in the CTEQ framework, arXiv:1509.00792.

[22] S. Atashbar Tehrani, Nuclear parton densities and their uncertainties at the next-to-leading order, Phys. Rev. C 86, 064301 (2012).

[23] S. A. Kulagin and R. Petti, Nuclear parton distributions and the Drell-Yan process, Phys. Rev. C 90, 045204 (2014).

[24] D. de Florian, R. Sassot, P. Zurita, and M. Stratmann, Global analysis of nuclear parton distributions, Phys. Rev. D 85, 074028 (2012).

[25] K. J. Eskola, H. Paukkunen, and C. A. Salgado, An improved global analysis of nuclear parton distribution functions including RHIC data, J. High Energy Phys. 07 (2008) 102.

[26] K. Kovarik, I. Schienbein, F. I. Olness, J. Y. Yu, C. Keppel, J. G. Morfin, J. F. Owens, and T. Stavreva, Nuclear Corrections in Neutrino-Nucleus DIS and Their Compatibility with Global NPDF Analyses, Phys. Rev. Lett. 106, 122301 (2011).

[27] K. J. Eskola, H. Paukkunen, and C. A. Salgado, EPS09: A new generation of NLO and LO nuclear parton distribution functions, J. High Energy Phys. 04 (2009) 065.

[28] M. Hirai, S. Kumano, and M. Miyama, Determination of nuclear parton distributions, Phys. Rev. D 64, 034003 (2001).

[29] M. Hirai, S. Kumano, and T.-H. Nagai, Nuclear parton distribution functions and their uncertainties, Phys. Rev. C 70, 044905 (2004).

[30] M. Hirai, S. Kumano, and T.-H. Nagai, Determination of nuclear parton distribution functions and their uncertainties in next-to-leading order, Phys. Rev. C 76, 065207 (2007).

[31] S. A. Tehrani, A. N. Khorramian, and A. Mirjalili, Nuclear parton densities and structure functions, Int. J. Mod. Phys. A 20, 1927 (2005).

[32] S. A. Tehrani, A. Mirjalili, and A. N. Khorramian, EMC effect and nuclear structure functions, Nucl. Phys. B, Proc. Suppl. 164, 30 (2007).

[33] S. A. Tehrani and A. N. Khorramian, QCD analysis for nuclear parton distributions in the next to leading order, eConf C 070910, 205 (2007).

[34] K. J. Eskola, V. J. Kolhinen, and C. A. Salgado, The scale dependent nuclear effects in parton distributions for practical applications, Eur. Phys. J. C 9, 61 (1999).

[35] K. J. Eskola, V. J. Kolhinen, and P. V. Ruuskanen, Scale evolution of nuclear parton distributions, Nucl. Phys. B535, 351 (1998).

[36] D. de Florian and R. Sassot, Nuclear parton distributions at next-to-leading order, Phys. Rev. D 69, 074028 (2004). 
[37] L. Frankfurt, V. Guzey, and M. Strikman, Leading twist nuclear shadowing phenomena in hard processes with nuclei, Phys. Rep. 512, 255 (2012).

[38] I. Schienbein, J. Y. Yu, K. Kovarik, C. Keppel, J. G. Morfin, F. Olness, and J. F. Owens, PDF nuclear corrections for charged and neutral current processes, Phys. Rev. D 80, 094004 (2009).

[39] D. de Florian, R. Sassot, M. Stratmann, and P. Zurita, Global Analysis of Nuclear PDFs, arXiv:1204.3797.

[40] R. D. Ball et al. (NNPDF Collaboration), Parton distributions for the LHC Run II, J. High Energy Phys. 04 (2015) 040.

[41] R. D. Ball, V. Bertone, S. Carrazza, C. S. Deans, L. Del Debbio, S. Forte, A. Guffanti, N. P. Hartland et al., Parton distributions with LHC data, Nucl. Phys. B867, 244 (2013).

[42] R. D. Ball, L. Del Debbio, S. Forte, A. Guffanti, J. I. Latorre, J. Rojo, and M. Ubiali, A first unbiased global NLO determination of parton distributions and their uncertainties, Nucl. Phys. B838, 136 (2010).

[43] R. D. Ball, V. Bertone, F. Cerutti, L. Del Debbio, S. Forte, A. Guffanti, J. I. Latorre, J. Rojo, and M. Ubiali, Impact of heavy quark masses on parton distributions and LHC phenomenology, Nucl. Phys. B849, 296 (2011).

[44] C. Loizides, First results from $\mathrm{p}-\mathrm{Pb}$ collisions at the LHC, EPJ Web Conf. 60, 06004 (2013).

[45] P. Bozek, Collective flow in $\mathrm{p}-\mathrm{Pb}$ and $\mathrm{d}-\mathrm{Pd}$ collisions at TeV energies, Phys. Rev. C 85, 014911 (2012).

[46] O. Busch (ALICE Collaboration), Jet structure in pp and $\mathrm{Pb}-\mathrm{Pb}$ collisions with the ALICE experiment, J. Phys. Conf. Ser. 589, 012003 (2015).

[47] T. Drozhzhova, Determination of classes of events in multiplicity and its relevance to centrality in high energy $\mathrm{Pb}-\mathrm{Pb}$ and $\mathrm{p}-\mathrm{Pb}$ collisions in different MC models, Proc. Sci., BaldinISHEPPXXII2015 (2015) 070.

[48] A. Dumitru, L. Frankfurt, L. Gerland, H. Stoecker, and M. Strikman, Nuclear broadening effects on hard prompt photons at relativistic energies, Phys. Rev. C 64, 054909 (2001).

[49] M. J. Tannenbaum, Highlights from BNL-RHIC 20112013, Int. J. Mod. Phys. A 29, 1430017 (2014).

[50] H. Paukkunen, Nuclear PDFs in the beginning of the LHC era, Nucl. Phys. A926, 24 (2014).

[51] R. Venugopalan, Why we need an Electron-Ion Collider, arXiv: 1507.08128.

[52] R. G. Milner, The Electron-Ion Collider science case, arXiv:1405.6930.

[53] E. C. Aschenauer et al., eRHIC Design study: An ElectronIon Collider at BNL, arXiv:1409.1633.

[54] E. Cruz-Alaniz and A. Valloni, LHeC accelerator development, Proc. Sci., DIS2014 (2014) 247.

[55] D. Boer et al., Gluons and the quark sea at high energies: distributions, polarization, tomography, arXiv:1108.1713.

[56] A. Polini (LHEC Collaboration), The large hadron electron collider at CERN, EPJ Web Conf. 73, 08005 (2014).

[57] P. Amaudruz et al. (New Muon Collaboration), A reevaluation of the nuclear structure function ratios for $\mathrm{D}, \mathrm{He}$, Li-6, C and Ca, Nucl. Phys. B441, 3 (1995).

[58] J. Gomez, R. G. Arnold, P. E. Bosted, C. C. Chang, A. T. Katramatou, G. G. Petratos, A. A. Rahbar, S. E. Rock et al., Measurement of the A-dependence of deep inelastic electron scattering, Phys. Rev. D 49, 4348 (1994).

[59] A. Bodek, N. Giokaris, W. B. Atwood, D. H. Coward, D. L. Dubin, M. Breidenbach, J. E. Elias, J. I. Friedman et al., A Comparison of the Deep Inelastic Structure Functions of Deuterium and Aluminum Nuclei, Phys. Rev. Lett. 51, 534 (1983).

[60] A. Bodek, N. Giokaris, W. B. Atwood, D. H. Coward, D. Sherden, D. L. Dubin, J. E. Elias, J. I. Friedman et al., Electron Scattering from Nuclear Targets and Quark Distributions in Nuclei, Phys. Rev. Lett. 50, 1431 (1983).

[61] S. Dasu, P. de Barbaro, A. Bodek, H. Harada, M. W. Krasny, K. Lang, E. M. Riordan, R. Arnold et al., Measurement of the Difference in $R=\sigma_{L} / \sigma_{T}$ and of $\sigma^{A} / \sigma^{D}$ in Deep-Inelastic $e-D, e-F e$, and $E-A u$ Scattering, Phys. Rev. Lett. 60, 2591 (1988).

[62] A. C. Benvenuti et al. (BCDMS Collaboration), Nuclear effects in deep inelastic muon scatter, Phys. Lett. B 189, 483 (1987).

[63] J. Ashman et al. (European Muon Collaboration), A measurement of the ratio of the nucleon structure function in copper and deuterium, Z. Phys. C 57, 211 (1993).

[64] J. Ashman et al. (European Muon Collaboration), Measurement of the ratios of deep inelastic muon-nucleus crosssections on various nuclei compared to deuterium, Phys. Lett. B 202, 603 (1988).

[65] M. R. Adams et al. (E665 Collaboration), Saturation of Shadowing at Very low $x_{\mathrm{Bj}}$, Phys. Rev. Lett. 68, 3266 (1992).

[66] M. R. Adams et al. (E665 Collaboration), Shadowing in inelastic scattering of muons on carbon, calcium and lead at low $x_{\mathrm{Bj}}$, Z. Phys. C 67, 403 (1995).

[67] J. Seely, A. Daniel, D. Gaskell, J. Arrington, N. Fomin, P. Solvignon, R. Asaturyan, F. Benmokhtar et al., New Measurements of the EMC Effect in Very Light Nuclei, Phys. Rev. Lett. 103, 202301 (2009).

[68] M. Arneodo et al. (New Muon. Collaboration), The structure function ratios $F_{2}^{C} / F_{2}^{D}$ and $F_{2}^{C} / F_{2}^{D}$ at small x, Nucl. Phys. B441, 12 (1995).

[69] M. Arneodo et al. (European Muon Collaboration), Measurements of the nucleon structure function in the range $0.002-\mathrm{GeV}^{2}<x<0.17-\mathrm{GeV}^{2}$ and $0.2-\mathrm{GeV}^{2}<$ $q^{2}<8-\mathrm{GeV}^{2}$ in deuterium, carbon and calcium, Nucl. Phys. B333, 1 (1990).

[70] G. Bari et al. (BCDMS Collaboration), A measurement of nuclear effects in deep inelastic muon scattering on deuterium, nitrogen and iron targets, Phys. Lett. 163B, 282 (1985).

[71] M. Arneodo et al. (New Muon Collaboration), The A dependence of the nuclear structure function ratios, Nucl. Phys. B481, 3 (1996).

[72] M. Arneodo et al. (New Muon Collaboration), The $Q^{2}$ dependence of the structure function ratio $F_{2}^{S n} / F_{2}^{C}$ and the difference $R^{S n}-R^{C}$ deep inelastic muon scattering, Nucl. Phys. B481, 23 (1996).

[73] K. Ackerstaff et al. (HERMES Collaboration), Nuclear effects on $R=\sigma_{L} / \sigma_{T}$ in deep-inelastic scattering, Phys. Lett. B 475, 386 (2000); 567, 339(E) (2003). 
[74] M. A. Vasilev et al. (FNAL E866 and NuSea Collaborations), Parton Energy Loss Limits and Shadowing in DrellYan Dimuon Production, Phys. Rev. Lett. 83, 2304 (1999).

[75] D. M. Alde, H. W. Baer, T. A. Carey, G. T. Garvey, A. Klein, C. Lee, M. J. Leitch, J. W. Lillberg et al., Nuclear Dependence of Dimuon Production at $800-\mathrm{GeV}$. FNAL-772 Experiment, Phys. Rev. Lett. 64, 2479 (1990).

[76] K. Rith, Present status of the EMC effect, arXiv: 1402.5000 .

[77] K. J. Eskola, Global analysis of nuclear PDFs-latest developments, Nucl. Phys. A910-911, 163 (2013).

[78] S. Kumano, Flavor asymmetry of anti-quark distributions in the nucleon, Phys. Rep. 303, 183 (1998).

[79] G. T. Garvey and J.-C. Peng, Flavor asymmetry of light quarks in the nucleon sea, Prog. Part. Nucl. Phys. 47, 203 (2001).

[80] A. Kusina, T. Stavreva, S. Berge, F. I. Olness, I. Schienbein, K. Kovarik, T. Jezo, J. Y. Yu, and K. Park, Strange quark PDFs and implications for Drell-Yan boson production at the LHC, Phys. Rev. D 85, 094028 (2012).

[81] L. L. Frankfurt, M. I. Strikman, and S. Liuti, Evidence for Enhancement of Gluon and Valence Quark Distributions in Nuclei from Hard Lepton Nucleus Processes, Phys. Rev. Lett. 65, 1725 (1990).

[82] A. Vogt, Efficient evolution of unpolarized and polarized parton distributions with QCD-PEGASUS, Comput. Phys. Commun. 170, 65 (2005).

[83] J. A. M. Vermaseren, A. Vogt, and S. Moch, The thirdorder QCD corrections to deep-inelastic scattering by photon exchange, Nucl. Phys. B724, 3 (2005).

[84] W. L. van Neerven and A. Vogt, NNLO evolution of deep inelastic structure functions: The nonsinglet case, Nucl. Phys. B568, 263 (2000).

[85] W. L. van Neerven and A. Vogt, NNLO evolution of deep inelastic structure functions: The singlet case, Nucl. Phys. B588, 345 (2000).

[86] F. James, CERN Program Library Long Writeup Report No. D506, 1994.

[87] A. D. Martin, R. G. Roberts, W. J. Stirling, and R. S. Thorne, Uncertainties of predictions from parton distributions. 1: Experimental errors, Eur. Phys. J. C 28, 455 (2003).

[88] J. Pumplin, D. Stump, R. Brock, D. Casey, J. Huston, J. Kalk, H. L. Lai, and W. K. Tung, Uncertainties of predictions from parton distribution functions. II. The Hessian method, Phys. Rev. D 65, 014013 (2001).

[89] S. T. Monfared, A. N. Khorramian, and S. A. Tehrani, A global analysis of diffractive events at HERA, J. Phys. G 39, 085009 (2012).

[90] F. Arbabifar, A. N. Khorramian, and M. Soleymaninia, QCD analysis of polarized DIS and the SIDIS asymmetry world data and light sea-quark decomposition, Phys. Rev. D 89, 034006 (2014).

[91] A. Accardi, N. Armesto, M. Botje, S. J. Brodsky, B. Cole, K. J. Eskola, G. I. Fai, L. Frankfurt et al., Hard probes in heavy ion collisions at the LHC: PDFs, shadowing and $p A$ collisions, arXiv:hep-ph/0308248.

[92] C. A. Salgado, J. Alvarez-Muniz, F. Arleo, N. Armesto, M. Botje, M. Cacciari, J. Campbell, C. Carli et al., Protonnucleus collisions at the LHC: Scientific opportunities and requirements, J. Phys. G 39, 015010 (2012).
[93] N. Armesto, J. Rojo, C. A. Salgado, and P. Zurita, Bayesian reweighting of nuclear PDFs and constraints from proton-lead collisions at the LHC, J. High Energy Phys. 11 (2013) 015.

[94] K. J. Eskola, H. Paukkunen, and C. A. Salgado, A perturbative QCD study of dijets in $\mathrm{p}+\mathrm{Pb}$ collisions at the LHC, J. High Energy Phys. 10 (2013) 213.

[95] H. Paukkunen, The LHC p + Pb run from the nuclear PDF perspective, Proc. Sci., DIS2014 (2014) 053.

[96] C. Loizides, First results on $\mathrm{p}-\mathrm{Pb}$ collisions from ALICE, Ann. Phys. (Amsterdam) 352, 41 (2015).

[97] S. Chatrchyan et al. (CMS Collaboration), Studies of dijet transverse momentum balance and pseudorapidity distributions in $\mathrm{pPb}$ collisions at $\sqrt{s_{\mathrm{NN}}}=5.02 \mathrm{TeV}$, Eur. Phys. J. C 74, 2951 (2014).

[98] R. Granier de Cassagnac (CMS Collaboration), CMS heavy-ion overview, Nucl. Phys. A931, 13 (2014).

[99] CMS Collaboration, https://twiki.cern.ch/twiki/bin/view/ CMSPublic/PhysicsResultsHIN.

[100] S. Salur (CMS Collaboration), A brief review of CMS jet measurements, J. Phys. Conf. Ser. 589, 012017 (2015).

[101] J. Adam et al. (ALICE Collaboration), Measurement of dijet $k_{\mathrm{T}}$ in $\mathrm{p}-\mathrm{Pb}$ collisions at $\sqrt{s_{\mathrm{NN}}}=5.02 \mathrm{TeV}$, Phys. Lett. B 746, 385 (2015).

[102] H. Paukkunen, Neutron skin and centrality classification in high-energy heavy-ion collisions at the LHC, Phys. Lett. B 745, 73 (2015).

[103] J. Adam et al. (ALICE Collaboration), Measurement of charged jet production cross sections and nuclear modification in $\mathrm{p}-\mathrm{Pb}$ collisions at $\sqrt{s_{\mathrm{NN}}}=5.02 \mathrm{TeV}$, Phys. Lett. B 749, 68 (2015).

[104] V. Khachatryan et al. (CMS Collaboration), Nuclear effects on the transverse momentum spectra of charged particles in $\mathrm{pPb}$ collisions at $\sqrt{s_{\mathrm{NN}}}=5.02 \mathrm{TeV}$, Eur. Phys. J. C 75, 237 (2015).

[105] H. Kim (CMS Collaboration), Open beauty measurements in $\mathrm{pPb}$ collisions with CMS, Nucl. Phys. A931, 558 (2014).

[106] B. De la Cruz (CMS Collaboration), Proceedings, 49th Rencontres de Moriond on QCD and High Energy (2014), pp. 329-332.

[107] M. Alvioli, B. A. Cole, L. Frankfurt, D. V. Perepelitsa, and M. Strikman, Evidence for $\mathrm{x}$-dependent proton color fluctuations in pA collisions at the LHC, arXiv:1409.7381 [Phys. Rev. D (to be Published)].

[108] R. A. Barbieri (CMS Collaboration), Studies of dijet and photon-jet properties in $\mathrm{pp}, \mathrm{pPb}$, and $\mathrm{PbPb}$ collisions with CMS, Nucl. Phys. A931, 465 (2014).

[109] Y. J. Lee (CMS Collaboration), Studies of dijet pseudorapidity distributions and transverse momentum balance in $\mathrm{pPb}$ collisions at $\sqrt{s_{N N}}=5.02 \mathrm{TeV}$, Ann. Phys. (Amsterdam) 352, 27 (2015).

[110] V. Khachatryan et al. (CMS Collaboration), Study of W boson production in $\mathrm{pPb}$ collisions at $\sqrt{s_{\mathrm{NN}}}=5.02 \mathrm{TeV}$, Phys. Lett. B 750, 565 (2015).

[111] S. Chatrchyan et al. (CMS Collaboration), Study of $W$ boson production in $\mathrm{PbPb}$ and $p p$ collisions at $\sqrt{s_{N N}}=2.76 \mathrm{TeV}$, Phys. Lett. B 715, 66 (2012).

[112] A. J. Zsigmond (CMS Collaboration), Z and W boson production in $\mathrm{pPb}$ collisions with CMS, Nucl. Phys. A931, 718 (2014). 
[113] P. Ru, B. W. Zhang, L. Cheng, E. Wang, and W. N. Zhang, Production of $Z^{0}$ and $W^{+} / W^{-}$in relativistic heavy-ion collisions at the LHC, J. Phys. G 42, 085104 (2015).

[114] S. Chatrchyan et al. (CMS Collaboration), Study of Z production in $\mathrm{PbPb}$ and pp collisions at $\sqrt{s_{\mathrm{NN}}}=2.76 \mathrm{TeV}$ in the dimuon and dielectron decay channels, J. High Energy Phys. 03 (2015) 022.

[115] N. Armesto, H. Paukkunen, J. M. Penín, C. A. Salgado, and P. Zurita, An analysis of the impact of LHC Run I proton-lead data on nuclear parton densities, arXiv:1512.01528.

[116] S. Chatrchyan et al. (CMS Collaboration), Study of Z Boson Production in $\mathrm{PbPb}$ Collisions at $\sqrt{s_{N N}}=2.76 \mathrm{TeV}$, Phys. Rev. Lett. 106, 212301 (2011).

[117] A. J. Zsigmond (CMS Collaboration), Study of Z boson production in $\mathrm{pp}, \mathrm{pPb}$ and $\mathrm{PbPb}$ collisions in CMS, J. Phys. Conf. Ser. 612, 012011 (2015).

[118] A. V. Baskakov, E. E. Boos, L. V. Dudko, I. P. Lokhtin, and A. M. Snigirev, Single top quark production in heavy ion collisions at the LHC, Phys. Rev. C 92, 044901 (2015).
[119] D. d'Enterria, K. Krajczar, and H. Paukkunen, Top-quark production in proton-nucleus and nucleus-nucleus collisions at LHC energies and beyond, Phys. Lett. B 746, 64 (2015).

[120] J. Arrington et al. (E906 Collaboration), Fermilab Projects doc No. 395, 2006, http:॥|projects-docdb.fnal.gov/cgi-bin/ ShowDocument?docid=395.

[121] P.E. Reimer, Opportunities with Drell-Yan scattering: Probing sea quarks in the nucleon and nuclei, Eur. Phys. J. A 31, 593 (2007).

[122] P. E. Reimer (Fermilab SeaQuest Collaboration), Opportunities with Drell-Yan scattering at Fermilab, J. Phys. Conf. Ser. 295, 012011 (2011).

[123] E. R. Cazaroto, F. Carvalho, V. P. Goncalves, and F. S. Navarra, Constraining the nuclear gluon distribution in eA processes at RHIC, Phys. Lett. B 669, 331 (2008).

[124] M. J. Tannenbaum, Hard-scattering and jets from RHIC to LHC: A critical review, Proc. Sci., LHC07 (2007) 004.

[125] S.S. Adler et al. (PHENIX Collaboration), Centrality Dependence of $\pi^{0}$ and $\eta$ Production at Large Transverse Momentum in $\sqrt{s_{N N}}=200 \mathrm{GeV} d+$ Au Collisions, Phys. Rev. Lett. 98, 172302 (2007). 